\title{
Naturalness in low-scale SUSY models and "non-linear" MSSM
}

\author{
I. Antoniadis ${ }^{1}$, E. M. Babalic ${ }^{2,3}$, D. M. Ghilencea ${ }^{1,2, a}$ \\ ${ }^{1}$ CERN Theory Division, 1211 Geneva 23, Switzerland \\ 2 Theoretical Physics Department, National Institute of Physics and Nuclear Engineering (IFIN-HH), MG-6 077125 Bucharest, Romania \\ ${ }^{3}$ Department of Mathematics and Natural Sciences, University of Craiova, 13 A. I. Cuza street, 200585 Craiova, Romania
}

Received: 17 July 2014 / Accepted: 31 August 2014 / Published online: 25 September 2014

(C) The Author(s) 2014. This article is published with open access at Springerlink.com

\begin{abstract}
In MSSM models with various boundary conditions for the soft breaking terms $\left(m_{\text {soft }}\right)$ and for a Higgs mass of $126 \mathrm{GeV}$, there is a (minimal) electroweak finetuning $\Delta \approx 800$ to 1000 for the constrained MSSM and $\Delta \approx 500$ for non-universal gaugino masses. These values, often regarded as unacceptably large, may indicate a problem of supersymmetry (SUSY) breaking, rather than of SUSY itself. A minimal modification of these models is to lower the SUSY breaking scale in the hidden sector $(\sqrt{f})$ to few $\mathrm{TeV}$, which we show to restore naturalness to more acceptable levels $\Delta \approx 80$ for the most conservative case of low $\tan \beta$ and ultraviolet boundary conditions as in the constrained MSSM. This is done without introducing additional fields in the visible sector, unlike other models that attempt to reduce $\Delta$. In the present case $\Delta$ is reduced due to additional (effective) quartic Higgs couplings proportional to the ratio $m_{\text {soft }} / \sqrt{f}$ of the visible to the hidden sector SUSY breaking scales. These couplings are generated by the auxiliary component of the goldstino superfield. The model is discussed in the limit its sgoldstino component is integrated out so this superfield is realized non-linearly (hence the name of the model) while the other MSSM superfields are in their linear realization. By increasing the hidden sector scale $\sqrt{f}$ one obtains a continuous transition for fine-tuning values, from this model to the usual (gravity mediated) MSSM-like models.
\end{abstract}

\section{Introduction}

If supersymmetry (SUSY) is realized in Nature, it should be broken at some high scale. A consequence of SUSY breaking is the existence of a Goldstone fermion - the goldstino-and its scalar superpartner, the sgoldstino. The goldstino becomes the longitudinal component of the gravitino which is rendered massive (super-Higgs mechanism), with a mass of order $f / M_{P}$ where $\sqrt{f}$ is the scale of spontaneous supersymme-

a e-mail: ghilencea@theory.nipne.ro try breaking in the hidden sector and $M_{P}$ is the Planck scale. Also, the sgoldstino can become massive and decouple at low energies. One interesting possibility is that $\sqrt{f} \ll M_{P}$, which represents the case of the so-called low-scale SUSY breaking models that we analyze in this work. Then the longitudinal gravitino component couplings which are those of the goldstino and proportional to $1 / \sqrt{f}$ [1-5] are much stronger than the couplings of the transverse gravitino component fields, which are Planck-scale suppressed. The latter vanish in the gravity-decoupled limit and one is left with a goldstino superfield besides the matter and vector superfields of the model. The gravitino is then very light, in the milli-eV range if SUSY breaking is in the multi-TeV region.

In this work we consider a variation of the minimal supersymmetric standard model (MSSM) called "non-linear MSSM" defined in [6] (see also [7-9]) in which $\sqrt{f}$ is a free parameter that can be as low as few times the scale of soft breaking terms in the visible sector, denoted generically $m_{\text {soft }}$. We assume that all fields beyond the MSSM spectrum (if any) are heavier than $\sqrt{f}$ (including the sgoldstino). Then, at energies of few TeV, $E \sim m_{\text {soft }}<\sqrt{f}$ we have the MSSM fields and the (non-linear) goldstino superfield $(X)$ coupled to them. The auxiliary component field $F_{X}$ (with $\left\langle F_{X}\right\rangle \sim-f$ ) of $X$ can mediate interactions $(\propto 1 / f)$ between the MSSM fields and generate sizeable effective couplings, in particular in the Higgs sector, if $\sqrt{f}$ is low (few TeV). The study of their implications for the electroweak (EW) fine-tuning is one main purpose of this work. This energy regime can be described by a non-linear goldstino superfield ${ }^{1}$ that satisfies $X^{2}=0$ [8-11]. This constraint decouples (integrates out) the scalar component of $X$ (sgoldstino), independent of the visible sector details (it depends only on the hidden sector [12-14]). The alternative case of a light sgoldstino, one that can mix with the Standard Model (SM) Higgs, was studied in $[7,15,16]$. At even lower energies, below the sparticle masses

${ }^{1}$ Hence the name of the model: "non-linear" MSSM. 
one is left with the goldstino fermion coupled to SM fields only, and all supermultiplets are realized non-linearly, i.e. all superpartners are integrated out.

However, with so far negative searches for supersymmetry at the TeV scale, the original motivation for SUSY, of solving the hierarchy problem, is sometimes questioned, since the stability at the quantum level of the hierarchy EW scale $\ll M_{P}$ becomes more difficult to respect. Indeed, the EW scale $v^{2}=-m^{2} / \lambda$, where $m$ is a combination of soft masses ( $\left.m_{\text {soft }}\right)$, therefore $m \sim \mathrm{TeV}$ and $\lambda \sim \mathcal{O}(1)$, an effective quartic Higgs coupling; with an increasing $m \sim m_{\text {soft }}$, it is more difficult to obtain $v=246 \mathrm{GeV}$. This tension is quantified by EW scale fine-tuning measures, hereafter denoted generically $\Delta$, with two examples being $\Delta_{m}, \Delta_{q}$ [17-20] (early studies in [21-25]) defined as

$$
\begin{aligned}
\Delta_{m}= & \max \left|\Delta_{\gamma^{2}}\right|, \quad \Delta_{q}=\left\{\sum_{\gamma} \Delta_{\gamma^{2}}^{2}\right\}^{1 / 2}, \\
& \times \text { with } \Delta_{\gamma^{2}} \equiv \frac{\partial \ln v^{2}}{\partial \ln \gamma^{2}} .
\end{aligned}
$$

$\Delta_{q}$ and $\Delta_{m}$ quantify the variation of $v$ under small relative variations of the ultraviolet (UV) parameters $\gamma$ that denote the SUSY breaking parameters and the (bare) higgsino mass $\left(\mu_{0}\right) . \Delta_{m, q}$ are regarded as intuitive measures of the success of SUSY as a solution to the hierarchy problem. For the constrained MSSM, $\gamma$ denotes the set: $m_{0}, m_{12}, \mu_{0}, A_{t}, B_{0}$. For the recently measured Standard Model-like Higgs mass $m_{h} \approx 126 \mathrm{GeV}$ [26-29], minimal values of $\Delta_{m, q}$ in the constrained MSSM are $\approx 800-1000$ [30], reduced to $\approx 500$ for non-universal boundary conditions for gauginos. These values are rather far from those regarded by theorists as more "acceptable" (but still subjective) of 10 to 100.

One can ask, however, what relevance such values of the EW fine-tuning have for the realistic character of a model and whether less subjective, model-independent bounds actually exist. Recent results [31-33] (based on previous [30,34-37]) suggest that there is an interesting link between the EW finetuning and the minimal value of chi-square $\left(\chi_{\min }^{2}\right)$ to fit the EW observables. Under the condition that motivated SUSY of fixing the EW scale $v=v(\gamma)$ to its value $(246 \mathrm{GeV})$ and with some simplifying assumptions it was found that there exists a model-independent upper bound $\Delta_{q} \ll \exp \left(n_{d f}\right)$ [31-33]; here $n_{d f}$ is the number of degrees of freedom of the model, $n_{d f}=n_{\mathcal{O}}-n_{p}$ with $n_{\mathcal{O}}$ the number of observables and $n_{p}$ the number of parameters. Generically, $n_{d f} \sim 10$ or so; see for example Table 1 in [32], depending on the boundary conditions of the MSSM-like model. This gives $\Delta_{q} \ll \exp 5 \approx 150$ or so. This is an estimate of the magnitude one should seek for $\Delta$ and supports the common view mentioned above that a tuning $\Delta_{q} \approx 100$ is "acceptable". It should be noted, however, that the nearly exponential depen- dence of minimal $\Delta_{m, q} \approx \exp \left(m_{h} / \mathrm{GeV}\right)$ noticed in [38-41] and the theoretical error of $2-3 \mathrm{GeV}$ of the Higgs mass [42$44]$ bring an error factor to the "acceptable" value of $\Delta$ as large as $\exp (2) \approx 7.4($ or $\exp (3) \approx 20)$. Therefore any value of $\Delta$ should be regarded with due care. Nevertheless, the above results tell us that a small $\Delta$ is preferable.

This view is further confirmed by a less conservative approach, which shows that there is also a link between the EW fine-tuning and the covariance matrix of a model $[45,46]$ in the basis of UV parameters $(\gamma)$. This matrix was shown [46] to automatically contain contributions due to the EW fine-tuning w.r.t. parameters $\gamma$ and, in particular, the trace of its inverse contains a contribution proportional to $\Delta_{q}$. As a result, imposing a fixed, s-standard deviation of the value of chi-square $\chi^{2}$ from its minimal value $\chi_{\min }^{2}$, i.e. $\delta \chi^{2} \leq s^{2}$ $\left(\chi^{2}=\chi_{\min }^{2}+\delta \chi^{2}\right)$, then requires in the loop order considered that $\Delta_{q}$ have an upper bound [46]. This is a modelindependent result and supports our motivation here of seeking models with low $\Delta$.

A very large EW fine-tuning, which increases further with negative searches for SUSY may suggest that we do not understand well the mechanism of SUSY breaking (assuming that SUSY exists not far above the TeV scale). This motivated us to consider the models with low SUSY breaking scale mentioned above and to evaluate their EW finetuning for the recently measured Higgs mass. (An early, preLHC study of other models with low SUSY scale is found in [47-49].) We examine the values of both $\Delta_{m}$ and $\Delta_{q}$ in the "non-linear MSSM" [6] which has a low scale of SUSY breaking, $\sqrt{f} \sim$ few TeV. The only difference of this model from the usual MSSM is present in the gravitino/goldstino and dark matter sectors. We show that this model can have a reduced fine-tuning compared to that in the MSSM-like models. The reduction is done without additional parameters or extra fields in the "visible" sector, which is unlike other models that reduce EW fine-tuning by enlarging the spectrum. Our results depend only on the ratio $m_{\text {soft }}^{2} / f$ of the SUSY breaking scale in the visible sector to that in the hidden sector. When $\sqrt{f}$ is low (few TeV) we are in the region of low-scale-SUSY breaking models (with light gravitino) while at large $\sqrt{f} \sim 10^{10} \mathrm{GeV}$ we recover the MSSM-like models. We thus have an interpolating parameter between these classes of models. The reason why EW fine-tuning is reduced is the additional quartic Higgs interactions mediated by the auxiliary component of the goldstino superfield, as mentioned earlier; these enhance the effective Higgs coupling $\lambda$ and even increase the Higgs mass already at tree level. We stress that this behavior is generic to low-scale SUSY models.

In the next section we review the model. In Sect. 3 we compute analytically the one-loop corrected Higgs mass including $\mathcal{O}\left(1 / f^{2}\right)$ corrections from effective operators generated by SUSY breaking. In Sect. 4 we compute at one 
loop $\Delta_{m, q}$ as functions of the SUSY breaking parameters and $\sqrt{f}$ and then present their numerical values in terms of the one-loop SM-like Higgs mass. For a most conservative case of low $\tan \beta$ and constrained MSSM boundary conditions for the soft terms, we find in "non-linear" MSSM an "acceptable" $\Delta_{m} \approx 80\left(\Delta_{q} \approx 120\right)$ for $\sqrt{f}=2.8 \mathrm{TeV}$ and $m_{h} \approx 126 \mathrm{GeV}$. This value of $\Delta$ can be reduced further for non-universal gaugino masses and is well below that in the constrained MSSM (for any $\tan \beta$ ) where $\Delta_{m, q} \sim 800-1000$ [30]. This reduction is done without enlarging the MSSM spectrum (for an example with additional massive singlets see $[50,51])$.

\section{The Lagrangian in "non-linear" MSSM}

The Lagrangian of the "non-linear MSSM" model can be written as [6-9]

$\mathcal{L}=\mathcal{L}_{0}+\mathcal{L}_{X}+\mathcal{L}_{1}+\mathcal{L}_{2}$

$\mathcal{L}_{0}$ is the usual MSSM SUSY Lagrangian which we write below to establish the notation:

$$
\begin{aligned}
\mathcal{L}_{0}= & \sum_{\Phi, H_{1,2}} \int \mathrm{d}^{4} \theta \Phi^{\dagger} e^{V_{i}} \Phi+\left\{\int \mathrm { d } ^ { 2 } \theta \left[\mu H_{1} H_{2}+H_{2} Q U^{c}\right.\right. \\
& \left.\left.+Q D^{c} H_{1}+L E^{c} H_{1}\right]+ \text { h.c. }\right\} \\
& +\sum_{i=1}^{3} \frac{1}{16 g_{i}^{2} \kappa} \int \mathrm{d}^{2} \theta \operatorname{Tr}\left[W^{\alpha} W_{\alpha}\right]_{i} \\
& + \text { h.c., } \Phi: Q, D^{c}, U^{c}, E^{c}, L
\end{aligned}
$$

$\kappa$ is a constant canceling the trace factor, and the gauge coupling is $g_{i}, i=1,2,3$ for $U(1)_{Y}, S U(2)_{L}$, and $S U(3)$, respectively. Further, $\mathcal{L}_{X}$ is the Lagrangian of the goldstino superfield $X=\left(\phi_{X}, \psi_{X}, F_{X}\right)$ that breaks SUSY spontaneously and whose Weyl component is "eaten" by the gravitino (super-Higgs effect $[52,53]) . \mathcal{L}_{X}$ can be written as $[8,9]$

$\mathcal{L}_{X}=\int \mathrm{d}^{4} \theta X^{\dagger} X+\left\{\int \mathrm{d}^{2} \theta f X+\right.$ h.c. $\}$ with $X^{2}=0$.

The otherwise interaction-free $\mathcal{L}_{X}$ when endowed with a constraint $X^{2}=0$ [8-11] describes (on-shell) the AkulovVolkov Lagrangian of the goldstino [54]; see also [55-61], with non-linear SUSY. The constraint has a solution $\phi_{X}=$ $\psi_{X} \psi_{X} /\left(2 F_{X}\right)$ that projects (integrates) out the sgoldstino field which becomes massive and is appropriate for a low energy description of SUSY breaking. Further, $\left\langle F_{X}\right\rangle \sim-f$ fixes the SUSY breaking scale $(\sqrt{f})$ and the breaking is transmitted to the visible sector by the couplings of $X$ to the MSSM superfields, to generate the usual SUSY breaking (effective) terms in $\mathcal{L}_{1}+\mathcal{L}_{2}$ (see below). These couplings are commonly parametrized (on-shell) in terms of the spurion field $S=m_{\text {soft }} \theta \theta$ where $m_{\text {soft }}$ is a generic notation for the soft masses (later denoted $m_{1,2,3}, m_{\lambda_{i}}$ ); however, this parametrization obscures the dynamics of $X$ (offshell effects) relevant below that generates additional Feynman diagrams mediated by $F_{X}$ (Fig. 1). Such effects are not seen in the leading order (in $1 / f$ ) in the spurion formalism. The off-shell couplings are easily recovered by the formal replacement $[8,9]$

$S \rightarrow \frac{m_{\mathrm{soft}}}{f} X$

In this way one obtains the SUSY breaking couplings that are indeed identical to those obtained by the equivalence theorem [1-5] from a theory with the corresponding explicit soft breaking terms and in which the goldstino fermion couples to the derivative of the supercurrent of the initial theory. These couplings are generated by the D-terms below:

$$
\begin{aligned}
\mathcal{L}_{1}= & \sum_{i=1,2} c_{i} \int \mathrm{d}^{4} \theta X^{\dagger} X H_{i}^{\dagger} e^{V_{i}} H_{i} \\
& +\sum_{\Phi} c_{\Phi} \int \mathrm{d}^{4} \theta X^{\dagger} X \Phi^{\dagger} e^{V} \Phi .
\end{aligned}
$$

and by the F-terms:

$$
\begin{aligned}
\mathcal{L}_{2}= & \sum_{i=1}^{3} \frac{1}{16 g_{i}^{2} \kappa} \frac{2 m_{\lambda_{i}}}{f} \int \mathrm{d}^{2} \theta X \operatorname{Tr}\left[W^{\alpha} W_{\alpha}\right]_{i} \\
& +c_{3} \int \mathrm{d}^{2} \theta X H_{1} H_{2}+\frac{A_{u}}{f} \int \mathrm{d}^{2} \theta X H_{2} Q U^{c} \\
& +\frac{A_{d}}{f} \int \mathrm{d}^{2} \theta X Q D^{c} H_{1}+\frac{A_{e}}{f} \int \mathrm{d}^{2} \theta X L E^{c} H_{1}+\text { h.c. }
\end{aligned}
$$

with

$$
\begin{aligned}
& c_{j}=-\frac{m_{j}^{2}}{f^{2}}, \quad j=1,2 ; \quad c_{3}=-\frac{m_{3}^{2}}{f}, \quad c_{\Phi}=-\frac{m_{\Phi}^{2}}{f^{2}} \\
& \Phi: Q, U^{c}, D^{c}, L, E^{c}
\end{aligned}
$$

In the UV one can eventually take $m_{\Phi}=m_{0}=m_{1}=m_{2}$, $m_{\lambda_{i}}=m_{12}(i=1,2,3)$ for all gaugino masses, $m_{3}^{2}=$ $B_{0} m_{0} \mu_{0}\left(\mu \equiv \mu_{0}\right.$ in the UV) and these define the "constrained" version of the "non-linear" MSSM, discussed later. For simplicity, Yukawa matrices are not displayed; to recover them just replace above any pair of fields $\phi_{Q} \phi_{U} \rightarrow \phi_{Q} \gamma_{u} \phi_{U}$, $\phi_{Q} \phi_{D} \rightarrow \phi_{Q} \gamma_{d} \phi_{D}, \phi_{L} \phi_{E} \rightarrow \phi_{L} \gamma_{e} \phi_{E}$; similar for the fermions and auxiliary fields, with $\gamma_{u, d, e} 3 \times 3$ matrices.

The total Lagrangian $\mathcal{L}$ defines the model discussed in detail in [6]. The only difference from the ordinary MSSM is in the supersymmetry breaking sector. In the calculation of the on-shell Lagrangian we restrict the calculations up to and including $1 / f^{2}$ terms. This requires solving for $F_{\phi}$ of matter fields up to and including $1 / f^{2}$ terms and for $F_{X}$ up to and including $1 / f^{3}$ terms (due to its leading contribution which 
is $-f)$. In this situation, in the final Lagrangian no kinetic mixing is present at the order used. ${ }^{2}$

\section{The Higgs masses at one loop in "non-linear" MSSM}

From the Lagrangian $\mathcal{L}$ one obtains the Higgs scalar potential of the model ${ }^{3}$ :

$$
\begin{aligned}
V= & \left(|\mu|^{2}+m_{1}^{2}\right)\left|h_{1}\right|^{2}+\left(|\mu|^{2}+m_{2}^{2}\right)\left|h_{2}\right|^{2} \\
& -\left(m_{3}^{2} h_{1} \cdot h_{2}+\text { h.c. }\right)+\left.\frac{1}{f^{2}}\left|m_{1}^{2}\right| h_{1}\right|^{2}+m_{2}^{2}\left|h_{2}\right|^{2} \\
& -\left.m_{3}^{2} h_{1} \cdot h_{2}\right|^{2}+\frac{g_{1}^{2}+g_{2}^{2}}{8}\left[\left|h_{1}\right|^{2}-\left|h_{2}\right|^{2}\right]^{2}+\frac{g_{2}^{2}}{2}\left|h_{1}^{\dagger} h_{2}\right|^{2} \\
& +\frac{\left.g_{1}^{2}+g_{2}^{2}\right)}{8} \delta\left|h_{2}\right|^{4}+\mathcal{O}\left(1 / f^{3}\right)
\end{aligned}
$$

with $h_{1} \cdot h_{2}=h_{1}^{0} h_{2}^{0}-h_{1}^{-} h_{2}^{+},\left|h_{1}\right|^{2}=\left|h_{1}^{0}\right|^{2}+\left|h_{1}^{-}\right|^{2},\left|h_{2}\right|^{2}=$ $\left|h_{2}^{0}\right|^{2}+\left|h_{2}^{+}\right|^{2}$.

What is interesting in the above Higgs potential is the presence of the first term in the second line of $V$, absent in MSSM, which is generated by the diagrams in Fig. 1. Therefore, quartic Higgs terms are generated by the dynamics of the goldstino superfield and are not captured by the usual spurion formalism in the MSSM. The impact of these terms for phenomenology is important and analyzed below, for when $\sqrt{f} \sim$ few TeV. When $\sqrt{f}$ is very large which is the case of MSSM-like models, these terms are negligible and thus not included by the spurion formalism. The ignored higher order terms $\mathcal{O}\left(1 / f^{3}\right)$ involve non-renormalizable $h_{1,2}^{6}$ interactions in $V$ and are not considered here. ${ }^{4}$ Finally, the radiatively corrected $m_{1,2,3}$ and $\mu$ in $V$ depend on the scale (hereafter denoted $t$ ) while the term $\delta\left|h_{2}\right|^{4}$ is generated at one loop by top-stop Yukawa couplings. We thus neglect other Yukawa couplings and our one-loop analysis is valid for low $\tan \beta$; including two-loop leading $\log$ effects $\delta$ is

$$
\begin{aligned}
\delta= & \frac{3 h_{t}^{4}}{g^{2} \pi^{2}}\left\{\ln \frac{M_{\tilde{t}}}{m_{t}}+\frac{X_{t}}{4}+\frac{1}{32 \pi^{2}}\left(3 h_{t}^{2}-16 g_{3}^{2}\right)\right. \\
& \left.\times\left(X_{t}+2 \ln \frac{M_{\tilde{t}}}{m_{t}}\right) \ln \frac{M_{\tilde{t}}}{m_{t}}\right\}
\end{aligned}
$$

\footnotetext{
$\overline{2}$ We stress that at energy scales below $m_{\text {soft }}$, similar constraints to that used for $X\left(X^{2}=0\right)$ can be applied to the MSSM superfields themselves and correspond to integrating out the massive superpartners $[8,9]$.

${ }^{3}$ In the standard notation for a two-Higgs doublet model $V=$ $\tilde{m}_{1}^{2}\left|h_{1}\right|^{2}+\tilde{m}_{2}^{2}\left|h_{2}\right|^{2}-\left(m_{3}^{2} h_{1} \cdot h_{2}+\right.$ h.c. $)+\frac{1}{2} \lambda_{1}\left|h_{1}\right|^{4}+\frac{1}{2} \lambda_{2}\left|h_{2}\right|^{4}+$ $\lambda_{3}\left|h_{1}\right|^{2}\left|h_{2}\right|^{2}+\lambda_{4}\left|h_{1} \cdot h_{2}\right|^{2}+\left[\frac{1}{2} \lambda_{5}\left(h_{1} \cdot h_{2}\right)^{2}+\lambda_{6}\left|h_{1}\right|^{2}\left(h_{1} \cdot h_{2}\right)+\right.$ $\lambda_{7}\left|h_{2}\right|^{2}\left(h_{1} \cdot h_{2}\right)+$ h.c. $]$ where $\tilde{m}_{1}^{2}=m_{1}^{2}+|\mu|^{2}, \tilde{m}_{2}^{2}=m_{2}^{2}+|\mu|^{2}$. $\lambda_{1} / 2=g^{2} / 8+m_{1}^{4} / f^{2}, \lambda_{2} / 2=g^{2}(1+\delta) / 8+m_{2}^{4} / f^{2}, \lambda_{3}=\left(g_{2}^{2}-\right.$ $\left.g_{1}^{2}\right) / 4+2 m_{1}^{2} m_{2}^{2} / f^{2}, \lambda_{4}=-g_{2}^{2} / 2+m_{3}^{4} / f^{2}, \lambda_{5}=0, \lambda_{6}=-m_{1}^{2} m_{3}^{2} / f^{2}$, $\lambda_{7}=-m_{2}^{2} m_{3}^{2} / f^{2}, g^{2}=g_{1}^{2}+g_{2}^{2}$.

${ }^{4}$ Effective operators in the Higgs sector in the SUSY context were discussed in the past $[49,62-69]$.
}

where

$$
X_{t} \equiv \frac{2\left(A_{t} m_{0}-\mu \cot \beta\right)^{2}}{M_{\tilde{t}}^{2}}\left(1-\frac{\left(A_{t} m_{0}-\mu \cot \beta\right)^{2}}{12 M_{\tilde{t}}^{2}}\right) \text {. }
$$

$M_{\tilde{t}}^{2}=m_{\tilde{t}_{1}} m_{\tilde{t}_{2}}$, and $g_{3}$ is the QCD coupling and $A_{t}$ is the dimensionless trilinear top coupling. ${ }^{5}$

The minimum conditions of the potential can be written

$$
-v^{2}=\frac{m^{2}}{\lambda}, \quad 2 \lambda \frac{\partial m^{2}}{\partial \beta}-m^{2} \frac{\partial \lambda}{\partial \beta}=0,
$$

with the notation ${ }^{6}$ :

$$
\begin{aligned}
m^{2} \equiv & \left(m_{1}^{2}+\mu^{2}\right) \cos ^{2} \beta+\left(m_{2}^{2}+\mu^{2}\right) \sin ^{2} \beta-m_{3}^{2} \sin 2 \beta, \\
\lambda \equiv & \frac{g_{1}^{2}+g_{2}^{2}}{8}\left[\cos ^{2} 2 \beta+\delta \sin ^{4} \beta\right] \\
& +\frac{1}{f^{2}}\left|m_{1}^{2} \cos ^{2} \beta+m_{2}^{2} \sin ^{2} \beta-(1 / 2) m_{3}^{2} \sin 2 \beta\right|^{2} .
\end{aligned}
$$

The correction to the effective quartic Higgs coupling $\lambda$, due to the soft terms $\left(m_{1,2,3}\right)$ has implications for the Higgs mass and EW fine-tuning. This positive correction could alleviate the relation between $v^{2}$ and $m^{2}$ : indeed, with $m \sim \mathcal{O}(1 \mathrm{TeV})$ and $\lambda \sim \mathcal{O}(1), v$ can only be of order $\mathcal{O}(1 \mathrm{TeV})$ as well. This brings about a tension between the EW scale and soft terms $(\sim m)$ which cannot easily be separated from each other; this tension is encoded by the EW fine-tuning measures, discussed in Sect. 4. Increasing $\lambda$ can alleviate this tension, with impact on the EW fine-tuning. Such a correction to $\lambda$ also arises in models with high scale breaking in the hidden sector, so it is present even in usual MSSM but is extremely small in that case since then $\sqrt{f} \sim 10^{10} \mathrm{GeV}$. Here we consider $\sqrt{f} \sim$ few $\mathrm{TeV}$, which is safely above the current lower bound of $\approx 700 \mathrm{GeV}[6,49,58,70]$.

The two minimum conditions of the scalar potential lead to

$$
\begin{aligned}
& m_{1}^{2}-m_{2}^{2}=\cot 2 \beta \\
& \quad \times\left[-m_{3}^{2}+\frac{f^{2}}{v^{2}} \frac{\left(-1+\sqrt{w_{0}}\right)\left[m_{3}^{2}+m_{Z}^{2} \sin 2 \beta\left(1-\left(\delta \sin ^{2} \beta\right) /(2 \cos 2 \beta)\right)\right]}{2 \mu^{2}+m_{Z}^{2}\left(\cos ^{2} 2 \beta+\delta \sin ^{4} \beta\right)-m_{3}^{2} \sin 2 \beta}\right], \\
& m_{1}^{2}+m_{2}^{2}=\frac{1}{\sin 2 \beta} \\
& \quad \times\left[m_{3}^{2}+\frac{f^{2}}{v^{2}} \frac{\left(-1+\sqrt{w}_{0}\right)\left[-m_{3}^{2}+\left(2 \mu^{2}+(\delta / 2) m_{Z}^{2} \sin ^{2} \beta\right) \sin 2 \beta\right]}{2 \mu^{2}+m_{Z}^{2}\left(\cos ^{2} 2 \beta+\delta \sin ^{4} \beta\right)-m_{3}^{2} \sin 2 \beta}\right]
\end{aligned}
$$

where

$$
w_{0} \equiv 1-\frac{v^{2}}{f^{2}}\left(4 \mu^{2}+2 m_{Z}^{2}\left(\cos ^{2} 2 \beta+\delta \sin ^{4} \beta\right)-2 m_{3}^{2} \sin 2 \beta\right) .
$$

\footnotetext{
$\overline{5 \text { More exactly }} A_{t}=A_{u} / m_{0}$ with $A_{u}$ as in Eq. (7).

${ }^{6}$ Also $\lambda \equiv\left(\lambda_{1} / 2\right) c_{\beta}^{4}+\left(\lambda_{2} / 2\right) s_{\beta}^{4}+\left(\lambda_{3}+\lambda_{4}+\lambda_{5}\right) s_{\beta}^{2} c_{\beta}^{2}+2 \lambda_{6} c_{\beta}^{3} s_{\beta}+$ $2 \lambda_{7} c_{\beta} s_{\beta}^{3}$ where we used the notation of footnote 3 and $s_{\beta}=\sin \beta, c_{\beta}=$ $\cos \beta, u \equiv \tan \beta=v_{2} / v_{1}, h_{i}=1 / \sqrt{2}\left(v_{i}+\tilde{h}_{i}\right), m_{Z}^{2}=\left(g_{1}^{2}+g_{2}^{2}\right) v^{2} / 4$.
} 


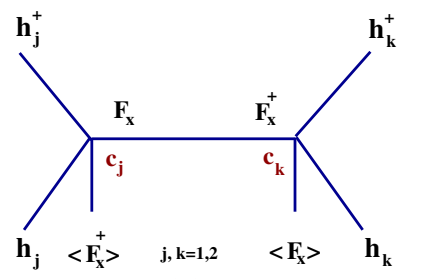

(a)

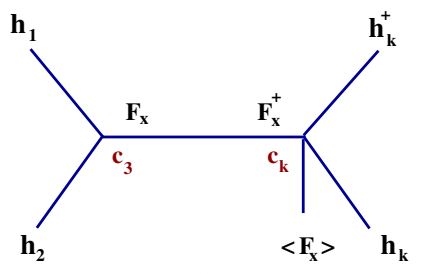

(b)

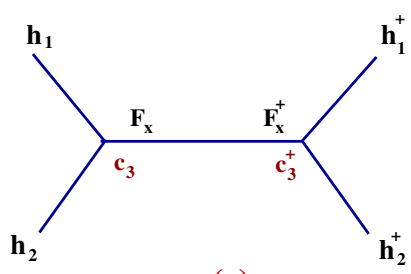

(c)
Fig. 1 The diagrams that generate the new quartic effective Higgs couplings in V, Eq. (9). The coefficients $c_{1,2,3}$ are generated by $\mathcal{L}_{1}, \mathcal{L}_{2} . F_{X}$ is the auxiliary component of $X$ that breaks SUSY. The left (right) diagrams are generated by $\mathrm{D}(\mathrm{F})$ terms in the action, while the middle one is

There is a second solution for $m_{1,2}^{2}$ at the minimum (with minus in front of $\sqrt{w}_{0}$ ) which, however, is not a perturbation of the MSSM solution and is not considered below (since it brings a shift proportional to $f$ of the soft masses, which invalidates the expansion in $m_{1,2}^{2} / f$ ).

The mass of the pseudoscalar Higgs is, including a oneloop correction (due to $\delta$ ):

$m_{A}^{2}=\frac{2 m_{3}^{2}}{\sin 2 \beta}\left\{\frac{3+\sqrt{w}_{0}}{4}-\frac{m_{3}^{2} v^{2}}{4 f^{2}} \sin 2 \beta\right\}$,

which can be expanded to $\mathcal{O}\left(1 / f^{3}\right)$ using the expression of $w_{0}$. For large $f$ one recovers its MSSM expression at one loop. Further, we computed the masses $m_{h, H}$ including the one-loop correction (due to $\delta$ ) to find

$m_{h, H}^{2}=\frac{1}{2}\left[m_{A}^{2}+m_{Z}^{2} \mp \sqrt{w}+\delta m_{Z}^{2} \sin ^{2} \beta\right]+\Delta m_{h, H}^{2}$

with upper (lower) sign corresponding to $m_{h}\left(m_{H}\right)$ and the correction $\Delta m_{h, H}^{2}=\mathcal{O}\left(1 / f^{2}\right)$ is

$$
\begin{aligned}
\Delta & m_{h, H}^{2}=\frac{v^{2}}{64 f^{2}}\left\{8 \left[8 \mu^{4}-2 m_{A}^{2} \mu^{2}+4 \mu^{2} m_{Z}^{2}+m_{Z}^{4}\right.\right. \\
& \left.+\left(2 m_{A}^{2} \mu^{2}+4 \mu^{2} m_{Z}^{2}+m_{Z}^{4}\right) \cos 4 \beta\right] \\
& -16 \delta m_{Z}^{2}\left[m_{A}^{2}-4 \mu^{2}+\left(m_{A}^{2}+2 m_{Z}^{2}\right) \cos 2 \beta\right] \sin ^{4} \beta \\
& +16 \delta^{2} m_{Z}^{4} \sin ^{6} \beta \pm(1 / \sqrt{w})\left[3 m_{A}^{6}-m_{A}^{4}\left(16 \mu^{2}+m_{Z}^{2}\right)\right. \\
& +4 m_{A}^{2}\left(16 \mu^{4}+4 \mu^{2} m_{Z}^{2}+m_{Z}^{4}\right)-8 m_{Z}^{4}\left(4 \mu^{2}+m_{Z}^{2}\right) \\
& -4\left[m_{A}^{6}+m_{A}^{4}\left(m_{Z}^{2}-4 \mu^{2}\right)-2 m_{A}^{2} m_{Z}^{2}\left(6 \mu^{2}+m_{Z}^{2}\right)\right. \\
& \left.+2 m_{Z}^{2}\left(8 \mu^{4}+4 \mu^{2} m_{Z}^{2}+m_{Z}^{4}\right)\right] \cos 4 \beta \\
& +m_{A}^{2}\left(m_{A}^{2}+m_{Z}^{2}\right)\left(m_{A}^{2}+4 m_{Z}^{2}\right) \cos 8 \beta \\
& +4 \delta m_{Z}^{2}\left[-m_{A}^{4}-2 m_{Z}^{4}+m_{A}^{2}\left(8 \mu^{2}+m_{Z}^{2}\right)\right. \\
& +\left(\left(m_{A}^{2}-4 \mu^{2}\right)^{2}-3\left(m_{A}^{2}-8 \mu^{2}\right) m_{Z}^{2}+7 m_{Z}^{4}\right) \cos 2 \beta \\
& +\left(m_{A}^{4}+\left(3 m_{A}^{2}-8 \mu^{2}\right) m_{Z}^{2}\right. \\
& \left.\left.-2 m_{Z}^{4}\right) \cos 4 \beta-\left(m_{A}^{4}+m_{A}^{2} m_{Z}^{2}-m_{Z}^{4}\right) \cos 6 \beta\right] \sin ^{2} \beta \\
& +16 \delta^{2} m_{Z}^{4}\left(m_{A}^{2}-4 \mu^{2}+3 m_{Z}^{2} \cos 2 \beta\right) \sin ^{6} \beta \\
& \left.\left.-16 \delta^{3} m_{Z}^{6} \sin ^{8} \beta\right]\right\}+\mathcal{O}\left(1 / f^{3}\right),
\end{aligned}
$$

a mixture of both. These interactions are important in low-scale SUSY breaking models while in the MSSM they are strongly suppressed since $\left\langle F_{X}\right\rangle$ is large)

with

$$
\begin{aligned}
w \equiv & \left(m_{A}^{2}+m_{Z}^{2}\right)^{2}-4 m_{A}^{2} m_{Z}^{2} \cos ^{2} 2 \beta+2 \delta\left(m_{A}^{2}-m_{Z}^{2}\right) \\
& \times m_{Z}^{2} \cos (2 \beta) \sin ^{2} \beta+\delta^{2} m_{Z}^{4} \sin ^{4} \beta .
\end{aligned}
$$

It is illustrative to take the limit of large $\tan \beta$ on $m_{h, H}^{2}$ with $m_{A}$ fixed. One finds

$$
\begin{aligned}
m_{h}^{2}= & {\left[(1+\delta) m_{Z}^{2}+\frac{v^{2}}{2 f^{2}}\left(2 \mu^{2}+(1+\delta) m_{Z}^{2}\right)^{2}\right.} \\
& \left.+\mathcal{O}\left(\cot ^{2} \beta\right)\right]+\mathcal{O}\left(1 / f^{3}\right) \\
m_{H}^{2}= & {\left[m_{A}^{2}+\mathcal{O}\left(\cot ^{2} \beta\right)\right]+\mathcal{O}\left(1 / f^{3}\right), }
\end{aligned}
$$

where we ignored the $\tan \beta$ dependence of $\delta$. Due to the $\mathcal{O}\left(\cot ^{2} \beta\right)$ suppression, Eq. (20) is valid even at smaller $\tan \beta \sim 10$. In this limit a significant increase of $m_{h}$ to 120 or even $126 \mathrm{GeV}$ is easily achieved, driven by classical effects alone with $\mu$ near $\mathrm{TeV}$ (and eventually small quantum corrections, $\delta \sim 0.5)$. Such an increase due to $\mu$ is thus of SUSY origin, even though the quartic Higgs couplings $\left(\mathcal{O}\left(1 / f^{2}\right)\right)$ giving this effect involved the soft masses $m_{1,2,3}$. These combined to give, at the $\mathrm{EW}$ minimum, the $\mu$-dependent increase in Eq. (20). For large $f$ one recovers the MSSM value of $m_{h, H}$, at one loop. Equations (17) and (18) are used in Sect. 4 to analyze the EW fine-tuning as a function of $m_{h}$.

\section{The electroweak scale fine-tuning}

\subsection{General results}

To compute the EW fine-tuning we use two definitions for it already shown in Introduction:

$$
\begin{gathered}
\Delta_{m}=\max \left|\Delta_{\gamma^{2}}\right|, \quad \Delta_{q}=\left\{\sum_{\gamma} \Delta_{\gamma^{2}}^{2}\right\}^{1 / 2}, \\
\text { with } \Delta_{\gamma^{2}} \equiv \frac{\partial \ln v^{2}}{\partial \ln \gamma^{2}},
\end{gathered}
$$

where $\gamma=m_{0}, m_{12}, A_{t}, B_{0}, \mu_{0}$ for the constrained "nonlinear" MSSM. In the following we evaluate $\Delta_{m}, \Delta_{q}$ at the 
one-loop level in our model. Using Eq. (12), which give $m^{2}=$ $m^{2}(\gamma, \beta)$ and $\lambda=\lambda(\gamma, \beta)$, one has a general result for $\Delta_{\gamma^{2}}$ which takes into account that $\tan \beta$ depends on $\gamma$ via the second min condition in Eq. (12). The result is $[47,48]$

$$
\begin{gathered}
\Delta_{\gamma^{2}}=-\frac{\gamma}{2 z}\left[\left(2 \frac{\partial^{2} m^{2}}{\partial \beta^{2}}+v^{2} \frac{\partial^{2} \lambda}{\partial \beta^{2}}\right)\left(\frac{\partial \lambda}{\partial \gamma}+\frac{1}{v^{2}} \frac{\partial m^{2}}{\partial \gamma}\right)\right. \\
\left.+\frac{\partial m^{2}}{\partial \beta} \frac{\partial^{2} \lambda}{\partial \beta \partial \gamma}-\frac{\partial \lambda}{\partial \beta} \frac{\partial^{2} m^{2}}{\partial \beta \partial \gamma}\right]
\end{gathered}
$$

where

$z \equiv \lambda\left(2 \frac{\partial^{2} m^{2}}{\partial \beta^{2}}+v^{2} \frac{\partial^{2} \lambda}{\partial \beta^{2}}\right)-\frac{v^{2}}{2}\left(\frac{\partial \lambda}{\partial \beta}\right)^{2}$.

Using these expressions, one obtains $\Delta_{m}$ and $\Delta_{q}$.

Let us first consider the limit of large $\tan \beta$, so the first relation in Eq. (12) becomes

$v^{2}=-\frac{2\left(m_{2}^{2}+\mu^{2}\right)}{(1+\delta)\left(g_{1}^{2}+g_{2}^{2}\right) / 4+2 m_{2}^{4} / f^{2}}+\mathcal{O}(\cot \beta)$,

which gives

$$
\Delta_{\gamma^{2}}=-\frac{\partial\left(m_{2}^{2}+\mu^{2}\right)}{\partial \ln \gamma} \frac{\left(1+2 v^{2} m_{2}^{2} / f^{2}\right)^{s}}{(1+\delta) m_{Z}^{2}+2 v^{2} m_{2}^{4} / f^{2}}+\mathcal{O}(\cot \beta),
$$

where $s=1$ if $\gamma \neq \mu_{0} ; s=0$ if $\gamma=\mu_{0}$, and $\mu, m_{2}$ are functions of the scale. ${ }^{7}$ If also $f$ is large, one recovers the MSSM corresponding expression (ignoring a $\tan \beta$ dependence of $\delta$ ):

$\Delta_{\gamma^{2}}^{0}=-\frac{\partial\left(m_{2}^{2}+\mu^{2}\right)}{\partial \ln \gamma} \frac{1}{(1+\delta) m_{Z}^{2}}+\mathcal{O}(\cot \beta)$,

which is interesting on its own. For the EW symmetry breaking to exist one must have $m_{2}^{2}+\mu^{2}<0$ and therefore $\Delta_{\gamma^{2}}$ of the "non-linear MSSM" is smaller than in the MSSM with similar UV boundary conditions for parameters $\gamma$. Indeed, in this case the ratio $r$ of $\Delta_{\gamma^{2}}$ to that in a MSSM-like model denoted $\Delta_{\gamma^{2}}^{0}$,

$r=\frac{\Delta_{\gamma^{2}}}{\Delta_{\gamma^{2}}^{0}}=\frac{\left(1+2 v^{2} m_{2}^{2} / f^{2}\right)^{s}(1+\delta) m_{Z}^{2}}{(1+\delta) m_{Z}^{2}+2 v^{2} m_{2}^{4} / f^{2}}+\mathcal{O}(\cot \beta)$,

is smaller than unity: $r \approx 1 / 2$ if $\delta \approx 0.8,\left|m_{2}^{2}\right| / f \approx 0.35$, and $r \approx 1 / 3$ if $\delta \approx 0.8,\left|m_{2}^{2}\right| / f \approx 0.5$ with $\sqrt{f}$ above the $\mathrm{TeV}$ scale (recall $\left|m_{2}^{2}\right| / f<1$ for convergence and $\delta \sim 0.5-$ 1). So for a large $\tan \beta$ the EW fine-tuning associated to each UV parameter is smaller relative to the MSSM and the same can then be said about overall $\Delta_{m}$ and $\Delta_{q}$. This reduction is actually more significant, since for the same point in the parameter space the Higgs mass is larger in

${ }^{7}$ As we shall detail shortly for the case of the constrained MSSM. the "non-linear" MSSM than in the MSSM alone, already at the tree level. Indeed, we saw in Eq. (20) that even in the absence of loop corrections one can easily achieve $m_{h} \approx 120 \mathrm{GeV}$, without the additional, significant finetuning "cost", present for $m_{h}>115 \mathrm{GeV}$ in the MSSM. This "cost" is $\Delta \sim \exp \left(\delta m_{h} / \mathrm{GeV}\right)$ due to loop corrections needed to increase $m_{h}$ by $\delta m_{g}$ in MSSM models; ${ }^{8}$ for the same $m_{h}$ the reduction is then expected to be by a factor $\Delta \sim \exp (120$ 115) $\sim 150$ relative to the constrained MSSM case. Then our $\Delta_{m, q}$ can be smaller by this factor and $r$ is also much smaller than unity when evaluated for the same $m_{h}$. Finally, fixing $m_{h}$ to its measured value is a very strong constraint on the parameter space, which, once satisfied, allows other EW constraints to be automatically respected [30], so this conclusion is unlikely to be affected by them.

Let us mention that in MSSM-like models the EW finetuning $\Delta$ is usually reduced as one increases $\tan \beta$ for a fixed $m_{h}$ (all the other parameters allowed to vary) [38-41]. This is because at large $\tan \beta$ additional Yukawa couplings effects (down sector) are enhanced and help the radiative EW symmetry breaking (thus reducing $\Delta$ ), while at small $\tan \beta$ this effect is suppressed [30]. The situation is similar to the above "non-linear" MSSM model. ${ }^{9}$

\subsection{The constrained "non-linear" MSSM}

The reduction of the EW fine-tuning in our model can be illustrated further by comparing it with that in the constrained MSSM (CMSSM) with universal UV scalar mass $m_{0}$ and gaugino mass $m_{12}$ and including only the top-stop Yukawa coupling correction. In that case one has

$m_{1}^{2}(t)=m_{0}^{2}+m_{12}^{2} \sigma_{1}(t), \quad \mu^{2}(t)=\mu_{0}^{2} \sigma_{8}^{2}(t)$,

$m_{2}^{2}(t)=m_{12}^{2} \sigma_{4}(t)+A_{t} m_{0} m_{12} \sigma_{5}(t)+m_{0}^{2} \sigma_{7}(t)-m_{0}^{2} A_{t}^{2} \sigma_{6}(t)$,

$m_{3}^{2}(t)=\mu_{0} m_{12} \sigma_{2}(t)+B_{0} m_{0} \mu_{0} \sigma_{8}(t)+\mu_{0} m_{0} A_{t} \sigma_{3}(t)$

where we made explicit the dependence of the soft masses $m_{1,2,3}$ and $\mu$ and of the coefficients $\sigma_{i}$ on the momentum scale $t=\ln \Lambda_{U V}^{2} / q^{2}$ induced by radiative corrections; $\sigma_{i}$ also depend on $\tan \beta$ and so do the soft masses. The high scale boundary conditions are chosen such as $\sigma_{1,2,3,4,5,6}(0)=0$, $\sigma_{7,8}(0)=1$ when quantum corrections are turned off. For $q^{2}=m_{Z}^{2}$ the values of $\sigma_{i}$ are given in the Appendix. These expressions are used in our numerical analysis below.

\footnotetext{
${ }^{8}$ For this exponential dependence on $m_{h}$ see figures 1 and 6 in the first reference in [38-41].

${ }^{9}$ As we show shortly for the conservative case of the constrained "nonlinear" MSSM, at small $\tan \beta$, fine-tuning is already acceptable, thus at larger $\tan \beta \Delta$ is expected to be similar or further reduced.
} 


\subsubsection{The large $\tan \beta$ case}

This regime was already discussed in the general case in Sect. 4.1. A numerical analysis of this case involves additional Yukawa couplings of the "down" sector not included in our $V$ and is beyond the goal of this paper. However, we can still provide further insight for the constrained "non-linear MSSM". From Eq. (25), one has

$$
\begin{aligned}
\Delta_{\mu_{0}^{2}}= & -\frac{2 \mu_{0}^{2} \sigma_{8}^{2}}{(1+\delta) m_{Z}^{2}+2 v^{2} m_{2}^{4} / f^{2}}+\mathcal{O}\left(\cot ^{2} \beta\right) \\
\Delta_{m_{0}^{2}}= & -\frac{m_{0}\left(1+2 v^{2} m_{2}^{2} / f^{2}\right)}{(1+\delta) m_{Z}^{2}+2 v^{2} m_{2}^{4} / f^{2}} \\
& \times\left(A_{t} \sigma_{5}-2 A_{t}^{2} m_{0} \sigma_{6}+2 m_{0} \sigma_{7}\right)+\mathcal{O}(\cot \beta) \\
\Delta_{m_{12}^{2}}= & -\frac{m_{12}\left(1+2 v^{2} m_{2}^{2} / f^{2}\right)}{(1+\delta) m_{Z}^{2}+2 v^{2} m_{2}^{4} / f^{2}} \\
& \times\left(2 m_{12} \sigma_{4}+A_{t} m_{0} \sigma_{5}\right)+\mathcal{O}(\cot \beta) \\
\Delta_{A_{t}^{2}}= & -\frac{A_{t}\left(1+2 v^{2} m_{2}^{2} / f^{2}\right)}{(1+\delta) m_{Z}^{2}+2 v^{2} m_{2}^{4} / f^{2}} \\
& \times\left(m_{12} \sigma_{5}-2 m_{0} A_{t} \sigma_{6}\right) m_{0}+\mathcal{O}(\cot \beta), \\
\Delta_{B_{0}^{2}}= & \mathcal{O}(\cot \beta) ;
\end{aligned}
$$

$m_{2}^{2}$ is given in Eq. (28) and, since $m_{2}^{2}<0$, the absolute values of the above $\Delta$ 's and then of $\Delta_{m, q}$ are smaller than those in the limit $f \rightarrow \infty$ when one recovers the constrained MSSM model (at large $\tan \beta$ ). So fine-tuning is reduced as already argued in the general discussion.

Turning off the quantum corrections to soft masses and $\mu$ $\left(\sigma_{1,2, \ldots, 6}=0, \sigma_{7,8}=1\right)$ and quartic coupling $(\delta=0)$, for large $f$, the above relations simplify to give for constrained MSSM

$\left|\Delta_{\gamma^{2}}\right|=\frac{2 \gamma^{2}}{m_{Z}^{2}}+\mathcal{O}(\cot \beta), \quad \gamma=m_{0}, \mu_{0}$

with the remaining expressions being $\mathcal{O}(\cot \beta)$. This also shows that in the constrained MSSM, the dominant contributions to fine-tuning (at classical level) are due to $m_{0}$ and $\mu_{0}$. In general, $\Delta_{m_{0}^{2}}$ is related to QCD effects that increase fine-tuning and dominates for $m_{h}>115 \mathrm{GeV}$ (fig.2 in the first reference in [38-41]). For TeV-valued $m_{0}=\mu_{0}=2$ $\mathrm{TeV}(\delta=0)$ one then has $\Delta_{q}=683$, which gives a good estimate of the value of fine-tuning in constrained MSSM. ${ }^{10}$ Equation (30) has close similarities to other fine-tuning measures defined in the literature such as $\Delta_{E W}$ of [71-73].

${ }^{10}$ For $m_{h} \approx 126 \mathrm{GeV}$, in constrained MSSM $\Delta_{m, q} \sim 800-1000$ [30].

\subsubsection{The small $\tan \beta$ case}

From Eqs. (21), (22), and (23) we find the following analytical results for $\Delta_{\gamma^{2}}$ at one-loop level:

$$
\begin{aligned}
& \Delta_{\mu_{0}^{2}}=-\frac{4}{D v^{2}}\left\{-2 f^{2} y_{1} \sin 2 \beta\left[(4+\delta) f^{2} m_{Z}^{2}\right.\right. \\
& +2 v^{2}\left(y_{1}^{2}+y_{2}^{2}\right)-2\left(\delta f^{2} m_{Z}^{2}+v^{2} y_{2} y_{3}\right) \cos 2 \beta \\
& \left.+\left[(4+\delta) f^{2} m_{Z}^{2}+2 v^{2} y_{1}^{2}\right] \cos 4 \beta-2 v^{2} y_{1} y_{2} \sin 4 \beta\right] \\
& +\left[\left[f^{2}\left(m_{Z}^{2} \delta+4 y_{2}\right)+2 v^{2} y_{2} y_{3}\right] \cos 2 \beta\right. \\
& -\left[(4+\delta) f^{2} m_{Z}^{2}+2 v^{2}\left(-y_{1}^{2}+y_{2}^{2}\right)\right] \cos 4 \beta \\
& \left.+2 y_{1}\left(4 f^{2}+v^{2} y_{3}-4 v^{2} y_{2} \cos 2 \beta\right) \sin 2 \beta\right] \\
& \times\left[8 f^{2} \mu_{0}^{2} \sigma_{8}^{2}+v^{2} y_{1}^{2}+y_{1}\left[-4 f^{2} \sin 2 \beta\right.\right. \\
& \left.\left.\left.+v^{2}\left(-y_{1} \cos 4 \beta-2 y_{3} \sin 2 \beta+y_{2} \sin 4 \beta\right)\right]\right]\right\} \text {, } \\
& \Delta_{m_{0}^{2}}=-\frac{4 f^{2} m_{0}}{D}\left\{4 \left[\left[f^{2}\left(m_{Z}^{2} \delta+4 y_{2}\right)+2 v^{2} y_{2} y_{3}\right] \cos 2 \beta\right.\right. \\
& -\left[(4+\delta) f^{2} m_{Z}^{2}+2 v^{2}\left(y_{2}^{2}-y_{1}^{2}\right)\right] \cos 4 \beta \\
& \left.+2 y_{1}\left[4 f^{2}+v^{2}\left(y_{3}-4 y_{2} \cos 2 \beta\right)\right] \sin 2 \beta\right] \\
& \times\left[v ^ { - 2 } \left[2 m_{0} \cos ^{2} \beta+y_{4} \sin ^{2} \beta\right.\right. \\
& \left.-\mu_{0}\left(A_{t} \sigma_{3}+B_{0} \sigma_{8}\right) \sin 2 \beta\right] \\
& +\left(1 / f^{2}\right)\left[2 m_{0} \cos ^{2} \beta-\mu_{0}\left(A_{t} \sigma_{3}+B_{0} \sigma_{8}\right)\right. \\
& \left.\times \cos \beta \sin \beta+y_{4} \sin ^{2} \beta\right] \\
& \left.\times\left(y_{3}-y_{2} \cos 2 \beta-y_{1} \sin 2 \beta\right)\right] \\
& +8\left(-2 y_{1} \cos 2 \beta+y_{2} \sin 2 \beta\right)\left[( 1 / 2 ) \left[\mu _ { 0 } \left(A_{t} \sigma_{3}\right.\right.\right. \\
& \left.\left.+B_{0} \sigma_{8}\right) \cos 2 \beta+\left(2 m_{0}-y_{4}\right) \sin 2 \beta\right] \\
& \times\left(y_{2} \cos 2 \beta-y_{3}+y_{1} \sin 2 \beta\right)-\left[2 m_{0} \cos ^{2} \beta\right. \\
& \left.-\mu_{0}\left(A_{t} \sigma_{3}+B_{0} \sigma_{8}\right)(1 / 2) \sin 2 \beta+y_{4} \sin ^{2} \beta\right] \\
& \left.\times\left(y_{1} \cos 2 \beta-y_{2} \sin 2 \beta\right)\right]+\left(1 / v^{2}\right)\left[2 \mu _ { 0 } \left(A_{t} \sigma_{3}\right.\right. \\
& \left.\left.+B_{0} \sigma_{8}\right) \cos 2 \beta+\left(2 m_{0}-y_{4}\right) \sin 2 \beta\right] \\
& \times\left[-2 f^{2} m_{Z}^{2}(-\delta+(4+\delta) \cos 2 \beta) \sin 2 \beta\right. \\
& +4 v^{2}\left(-y_{3}+y_{2} \cos 2 \beta+y_{1} \sin 2 \beta\right)\left(y_{1} \cos 2 \beta\right. \\
& \left.\left.\left.-y_{2} \sin 2 \beta\right)\right]\right\} \text {, }
\end{aligned}
$$

and

$$
\begin{aligned}
& \Delta_{m_{12}^{2}}=\frac{-4 f^{2} m_{12}}{D}\left\{4 \left[\left[f^{2}\left(m_{Z}^{2} \delta+4 y_{2}\right)+2 v^{2} y_{2} y_{3}\right] \cos 2 \beta\right.\right. \\
& -\left[(4+\delta) f^{2} m_{Z}^{2}+2 v^{2}\left(y_{2}^{2}-y_{1}^{2}\right)\right] \\
& \left.\quad \times \cos 4 \beta+2 y_{1}\left(4 f^{2}+v^{2} y_{3}-4 v^{2} y_{2} \cos 2 \beta\right) \sin 2 \beta\right] \\
& \quad \times\left[\frac { 1 } { v ^ { 2 } } \left[2 m_{12} \sigma_{1} \cos ^{2} \beta-\mu_{0} \sigma_{2} \sin 2 \beta\right.\right.
\end{aligned}
$$


$\left.+\left(2 m_{12} \sigma_{4}+A_{t} m_{0} \sigma_{5}\right) \sin ^{2} \beta\right]+\left(1 / f^{2}\right)$

$\times\left[2 m_{12} \sigma_{1} \cos ^{2} \beta-(1 / 2) \mu_{0} \sigma_{2} \sin 2 \beta\right.$

$\left.\left.+\left(2 m_{12} \sigma_{4}+A_{t} m_{0} \sigma_{5}\right) \sin ^{2} \beta\right]\left(y_{3}-y_{2} \cos 2 \beta-y_{1} \sin 2 \beta\right)\right]$

$+8\left(y_{2} \sin 2 \beta-2 y_{1} \cos 2 \beta\right)$

$\times\left[(1 / 2)\left[\mu_{0} \sigma_{2} \cos 2 \beta+\left(2 m_{12}\left(\sigma_{1}-\sigma_{4}\right)-A_{t} m_{0} \sigma_{5}\right) \sin 2 \beta\right]\right.$

$\times\left(-y_{3}+y_{2} \cos 2 \beta+y_{1} \sin 2 \beta\right)$

$-\left[2 m_{12} \sigma_{1} \cos ^{2} \beta-\frac{1}{2} \mu_{0} \sigma_{2} \sin 2 \beta\right.$

$\left.\left.+\left(2 m_{12} \sigma_{4}+A_{t} m_{0} \sigma_{5}\right) \sin ^{2} \beta\right]\left(y_{1} \cos 2 \beta-y_{2} \sin 2 \beta\right)\right]$

$+\left(1 / v^{2}\right)\left[2 \mu_{0} \sigma_{2} \cos 2 \beta+\left[2 m_{12}\left(\sigma_{1}-\sigma_{4}\right)-A_{t} m_{0} \sigma_{5}\right] \sin 2 \beta\right]$

$\times\left[-2 f^{2} m_{Z}^{2}(-\delta+(4+\delta) \cos 2 \beta) \sin 2 \beta\right.$

$+4 v^{2}\left(-y_{3}+y_{2} \cos 2 \beta+y_{1} \sin 2 \beta\right)$

$\left.\left.\times\left(y_{1} \cos 2 \beta-y_{2} \sin 2 \beta\right)\right]\right\}$,

and

$\Delta_{A_{t}^{2}}=\frac{-4 A_{t}}{D}\left\{8 f^{2}\left(y_{2} \sin 2 \beta-2 y_{1} \cos 2 \beta\right)\right.$

$\times\left[\left(m_{0} / 2\right)\left(\mu_{0} \sigma_{3} \cos 2 \beta+\left(2 A_{t} m_{0} \sigma_{6}-m_{12} \sigma_{5}\right) \sin 2 \beta\right)\right.$

$\times\left(-y_{3}+y_{2} \cos 2 \beta+y_{1} \sin 2 \beta\right)+m_{0} \sin \beta$

$\times\left[\mu_{0} \sigma_{3} \cos \beta+\left(-m_{12} \sigma_{5}+2 A_{t} m_{0} \sigma_{6}\right) \sin \beta\right]$

$\left.\times\left(y_{1} \cos 2 \beta-y_{2} \sin 2 \beta\right)\right]+\left(f^{2} / v^{2}\right) m_{0}\left[2 \mu_{0} \sigma_{3} \cos 2 \beta\right.$

$\left.+\left(-m_{12} \sigma_{5}+2 A_{t} m_{0} \sigma_{6}\right) \sin 2 \beta\right]$

$\times\left[-2 f^{2} m_{Z}^{2}[-\delta+(4+\delta) \cos 2 \beta] \sin 2 \beta\right.$

$+4 v^{2}\left(-y_{3}+y_{2} \cos 2 \beta+y_{1} \sin 2 \beta\right)$

$\left.\times\left(y_{1} \cos 2 \beta-y_{2} \sin 2 \beta\right)\right]-\left(4 / v^{2}\right) m_{0} \sin \beta$

$\times\left[\left(f^{2}\left(\delta m_{Z}^{2}+4 y_{2}\right)+2 v^{2} y_{2} y_{3}\right) \cos 2 \beta\right.$

$-\left[f^{2} m_{Z}^{2}(4+\delta)+2 v^{2}\left(-y_{1}^{2}+y_{2}^{2}\right)\right] \cos 4 \beta$

$\left.+2 y_{1}\left(4 f^{2}+v^{2} y_{3}-4 v^{2} y_{2} \cos 2 \beta\right) \sin 2 \beta\right]$

$\times\left[\mu_{0} \sigma_{3} \cos \beta\left[2 f^{2}+v^{2} y_{3}-v^{2}\left(y_{2} \cos 2 \beta+y_{1} \sin 2 \beta\right)\right]\right.$

$+\left(m_{12} \sigma_{5}-2 A_{t} m_{0} \sigma_{6}\right) \sin \beta$

$\left.\left.\times\left[-f^{2}-v^{2} y_{3}+v^{2}\left(y_{2} \cos 2 \beta+y_{1} \sin 2 \beta\right)\right]\right]\right\}$.

Finally

$$
\begin{aligned}
& \Delta_{B_{0}^{2}}=-\frac{8 B_{0} m_{0} \mu_{0} \sigma_{8}}{D}\left\{\frac { \operatorname { s i n } 2 \beta } { v ^ { 2 } } \left[\left(f^{2}\left(\delta m_{Z}^{2}+4 y_{2}\right)\right.\right.\right. \\
& \left.+2 v^{2} y_{2} y_{3}\right) \cos 2 \beta-\left[(4+\delta) f^{2} m_{Z}^{2}\right. \\
& \left.+2 v^{2}\left(-y_{1}^{2}+y_{2}^{2}\right)\right] \cos 4 \beta+2 y_{1}\left(4 f^{2}+v^{2} y_{3}\right. \\
& \left.\left.-4 v^{2} y_{2} \cos 2 \beta\right) \sin 2 \beta\right]\left[-2 f^{2}-v^{2} y_{3}\right. \\
& \left.+v^{2}\left(y_{2} \cos 2 \beta+y_{1} \sin 2 \beta\right)\right]+\frac{f^{2}}{v^{2}} \cos 2 \beta
\end{aligned}
$$

$\times\left[-2 f^{2} m_{Z}^{2}[-\delta+(4+\delta) \cos 2 \beta] \sin 2 \beta+4 v^{2}\right.$

$\left.\times\left(-y_{3}+y_{2} \cos 2 \beta+y_{1} \sin 2 \beta\right)\left(y_{1} \cos 2 \beta-y_{2} \sin 2 \beta\right)\right]$

$-2 f^{2}\left(2 y_{1} \cos 2 \beta-y_{2} \sin 2 \beta\right)$

$\left.\times\left(-y_{3} \cos 2 \beta+y_{2} \cos 4 \beta+y_{1} \sin 4 \beta\right)\right\}$.

The denominator $D$ used in the above formulas is

$$
\begin{aligned}
D \equiv & 2 f^{2}\left[\left[f^{2}\left(m_{Z}^{2} \delta+4 y_{2}\right)+2 v^{2} y_{2} y_{3}\right] \cos 2 \beta\right. \\
& -\left[(4+\delta) f^{2} m_{Z}^{2}+2 v^{2}\left(y_{2}^{2}-y_{1}^{2}\right)\right] \cos 4 \beta \\
& \left.+2 y_{1}\left(4 f^{2}+v^{2} y_{3}-4 v^{2} y_{2} \cos 2 \beta\right) \sin 2 \beta\right] \\
& \times\left[8\left(m_{Z}^{2} / v^{2}\right)\left(\cos ^{2} 2 \beta+\delta \sin ^{4} \beta\right)+\left(4 / f^{2}\right)\left(-y_{3}\right.\right. \\
& \left.\left.+y_{2} \cos 2 \beta+y_{1} \sin 2 \beta\right)^{2}\right]-\left(1 / v^{2}\right)\left[-4 v^{2}\left(-y_{3}\right.\right. \\
& \left.+y_{2} \cos 2 \beta+y_{1} \sin 2 \beta\right)\left(y_{1} \cos 2 \beta\right. \\
& \left.\left.-y_{2} \sin 2 \beta\right)+f^{2} m_{Z}^{2}(-2 \delta \sin 2 \beta+(4+\delta) \sin 4 \beta)\right]^{2} .
\end{aligned}
$$

In the above expressions we introduced the notations:

$$
\begin{aligned}
& y_{1} \equiv \mu_{0}\left(m_{12} \sigma_{2}+A_{t} m_{0} \sigma_{3}+B_{0} m_{0} \sigma_{8}\right) \\
& y_{2} \equiv-m_{12}^{2}\left(\sigma_{1}-\sigma_{4}\right)-m_{0}\left(m_{0}-A_{t} m_{12} \sigma_{5}+A_{t}^{2} m_{0} \sigma_{6}-m_{0} \sigma_{7}\right) \\
& y_{3} \equiv y_{2}+2 \sigma_{1} m_{12}^{2}+2 m_{0}^{2} \\
& y_{4} \equiv A_{t} m_{12} \sigma_{5}-2 A_{t}^{2} m_{0} \sigma_{6}+2 m_{0} \sigma_{7}
\end{aligned}
$$

The expressions for $\Delta_{\gamma^{2}}$ simplify considerably if one turns off the quantum corrections to the soft terms $\left(\sigma_{1,2, \ldots, 6}=0\right.$, $\left.\sigma_{7,8}=1\right)$. We checked that in the limit of large $f, \Delta_{\gamma^{2}}$ recover the analytical results for fine-tuning at one loop found in [62] for the constrained MSSM (plus corrections $\left.\mathcal{O}\left(1 / f^{2}\right)\right)$. One also recovers from the above expressions for $\Delta_{\gamma^{2}}$ the results in Eq. (29).

\subsection{Numerical results}

Using the results in Eqs. (31) to (37) we evaluated $\Delta_{m}$ and $\Delta_{q}$ for fixed values of the SUSY breaking scale in the hidden sector $\sqrt{f}$ for $\tan \beta \leq 10$, subject to the EW constraints (for a discussion of these, see [30]). Note that imposing the Higgs mass range of $126 \pm$ (2 to 3 ) GeV (to allow for the theoretical error [42-44]) automatically respects these constraints [30]. For a rapid convergence of the perturbative expansion in $1 / f$ of the Lagrangian we demanded that $m_{\text {soft }}^{2} / f<1 / 4$, 


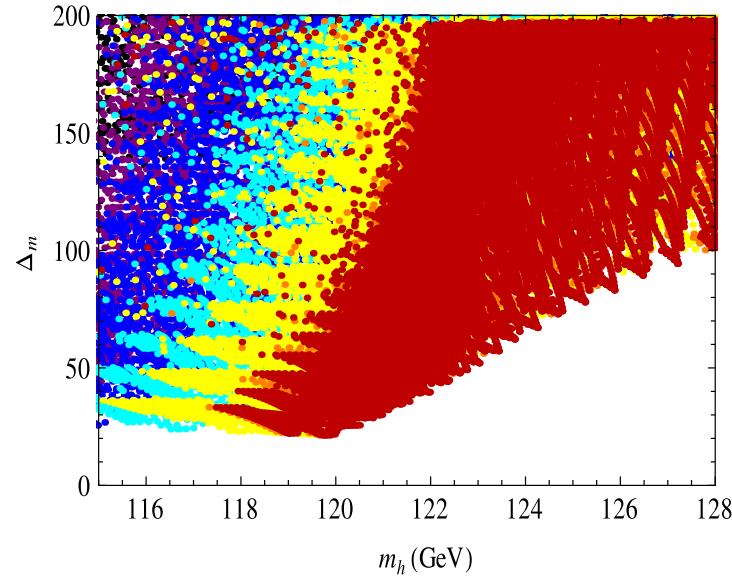

Fig. 2 The EW fine-tuning $\Delta_{m}$ (left) and $\Delta_{q}$ (right) as functions of the SM-like Higgs mass $m_{h}$ (in $\mathrm{GeV}$ ), all evaluated at one loop, for $\tan \beta \leq 10$. These plots have a fixed value $\sqrt{f}=2.8 \mathrm{TeV}$ of the SUSY breaking scale and $\tan \beta$ increases from left $(\tan \beta \leq 2.5)$ to right ( $\tan \beta=10)$ as shown by different colors: black/leftmost region: $\tan \beta \leq 2.5$; purple: $2.5 \leq \tan \beta \leq 4$; blue: $4 \leq \tan \beta \leq 4.5$; cyan: $4.5 \leq \tan \beta \leq 5.5$; yellow: $5.5 \leq \tan \beta \leq 9.5$; red/rightmost region:

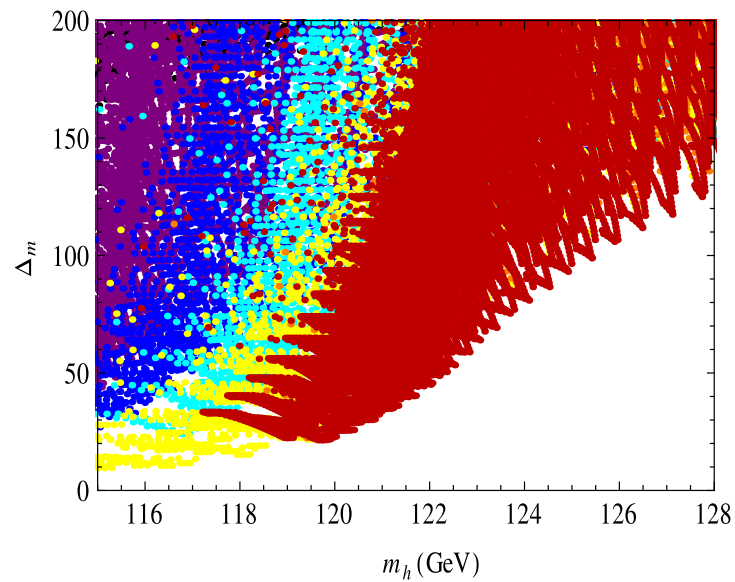

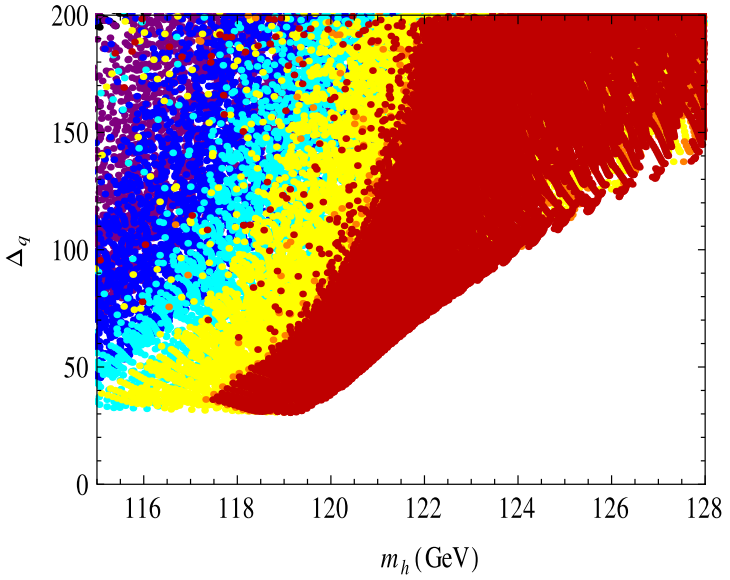

$\tan \beta=10$ (a larger $\tan \beta$ region is on top of that of smaller $\tan \beta$ ). For $m_{h}=126 \mathrm{GeV}$, minimal $\Delta_{m} \approx 80$ and $\Delta_{q} \approx 120$, while in the corresponding constrained MSSM minimal values (for $\tan \beta<55$ ), $\Delta_{m} \sim \Delta_{q} \approx 800-1000$, too large to be shown here; for details see figures 1-8 in [30]. The wide range of values for $m_{h}$ was chosen only to display the $\tan \beta$ dependence and to allow for the $2-3 \mathrm{GeV}$ theoretical error of $m_{h}[42-44]$

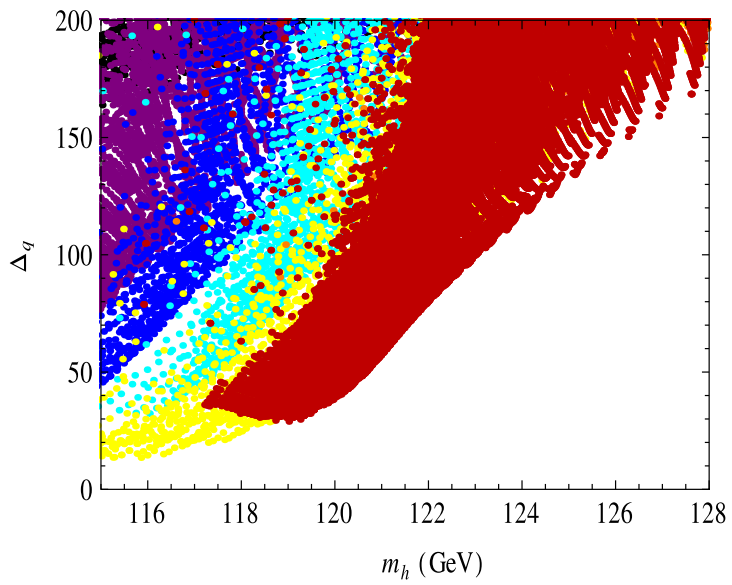

Fig. $3 \Delta_{m}$ (left) and $\Delta_{q}$ (right), with similar considerations as for Fig. 2 but with $\sqrt{f}=3.2 \mathrm{TeV}$. In this case, minimal $\Delta_{m}=105$ and $\Delta_{q}=145$ for $m_{h}=126 \mathrm{GeV}$

where $m_{\text {soft }}$ stands for SUSY breaking terms. ${ }^{11}$ The results are shown in Figs. 2, 3, and 4.

For $m_{h}=126 \mathrm{GeV}$ we find minimal values of $\Delta_{m} \approx 80$ and $\Delta_{q} \approx 120$ for $\sqrt{f}=2.8 \mathrm{TeV}$ (Fig. 2) and $\Delta_{m} \approx 105$ and $\Delta_{q} \approx 145$ for $\sqrt{f}=3.2 \mathrm{TeV}$ (Fig. 3). These values of $\sqrt{f}$ are well above the current lower bound of $\approx 700 \mathrm{GeV}$ $[6,49,58,70]$. As one increases $\tan \beta$ for a given $m_{h}, \Delta_{m}$ or $\Delta_{q}$ decreases, as shown by the color encoding corresponding to fixed $\tan \beta$ in Figs. 2 and 3; this is also valid in the MSSM as

\footnotetext{
11 This is a conservative bound, since in the potential (Eq. (9)) and in the Higgs mass of Eqs. (18), (20) the leading corrections are actually of the (higher) order $\mathcal{O}\left(m_{\text {soft }}^{4} / f^{2}\right)$ (we ignore the $\mathcal{O}\left(m_{\text {soft }}^{6} / f^{3}\right) \leq(1 / 4)^{3} \sim$ $1.5 \%$ or about $2 \mathrm{GeV}$ to the Higgs mass). Similar for the fine-tuning $\Delta$; see for example Eq. (29) or the exact results in Sect. 4.2.2, where the leading terms are $\mathcal{O}\left(1 / f^{2}\right)$.
}

seen in Figures 3, 4, 5 in the first reference in [38-41]. These values for fine-tuning are already "acceptable" and significantly below the minimal values in the constrained MSSM where for $m_{h} \approx 126 \mathrm{GeV}, \Delta_{m, q} \approx 800-1000$, see Figures $1-$ 8 in [30], obtained after scanning over all $2 \leq \tan \beta \leq 55$.

The reduced values of $\Delta_{m}$ and $\Delta_{q}$ are due to the fact that $m_{h}$ is significantly above that of the constrained MSSM already at the classical level, see Eqs. (17) to (20) for $\delta=0$, where values of $120-126 \mathrm{GeV}$ are easily achieved, so only very small quantum corrections are actually needed (unlike in the MSSM). This is a consequence of the (classically) increased effective quartic Higgs coupling. Also notice that minimal values of $\Delta_{m}$ and $\Delta_{q}$ have a similar dependence on $m_{h}$ and are only mildly different in size, as also noticed for the MSSM [30]. 


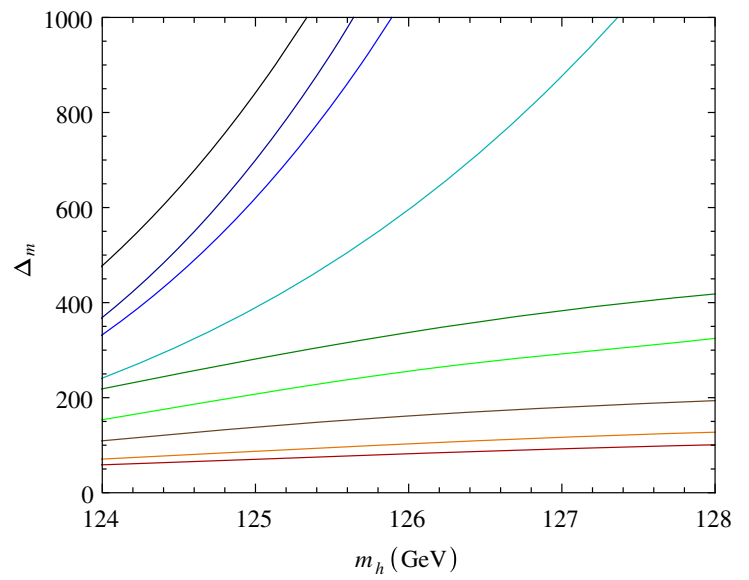

Fig. 4 The dependence of minimal $\Delta_{m}$ (left) and $\Delta_{q}$ (right) on $m_{h}$ $(\mathrm{GeV})$ for different $\sqrt{f}$, for fixed $\tan \beta=10$ with the other parameters allowed to vary. We allowed a $\pm 2 \mathrm{GeV}$ (theoretical) error for $m_{h}$ [42-44] about the central value of $126 \mathrm{GeV}$. For a fixed $m_{h}$ the minimal values of $\Delta_{m}, \Delta_{q}$ increase as we increase $\sqrt{f}$ from the lowest to the top curve, in this order: $2.8 \mathrm{TeV}$ (the lower/red curve), $3.2 \mathrm{TeV}$ (orange),

In Fig. 4 we presented the minimal values of $\Delta_{m}$ and $\Delta_{q}$ as functions of $m_{h}$ for fixed $\tan \beta=10$ for different values of the SUSY breaking scale from $\sqrt{f}=2.8 \mathrm{TeV}$ to 8.7 TeV. When increasing $\sqrt{f}$ to larger values, in the region above $10 \mathrm{TeV}$, the effects of the additional quartic terms in the scalar Higgs potential are rapidly suppressed and one recovers the usual constrained MSSM-like scenario with similar UV boundary conditions, with larger fine-tuning for the same $m_{h}$ and with minimal $\Delta_{q, m} \sim \exp \left(m_{h} / \mathrm{GeV}\right.$ ) (see the top curves in Fig. 4). This exponential behavior is characteristic for MSSM-like models due to (large) quantum corrections to the Higgs mass [38-41]. Relaxing the UV universality boundary condition for the gaugino masses reduces $\Delta_{m, q}$ further, similar to the MSSM $[23,30,74,75]$, by a factor of $\approx 2$ from the values given by the curves in Fig. 4 . Thus, values of $\sqrt{f}$ of up to 5-6 TeV can still give an EW fine-tuning of about $\sim 100$, for the low $\tan \beta$ regime considered here.

The case of constrained "non-linear" MSSM at small $\tan \beta \leq 10$, for which we found "acceptable" values for $\Delta_{m, q}$, is the most conservative scenario. We saw in Figs. 2 and 3 that for the same $m_{h}$ a larger $\tan \beta$ reduces fine-tuning and this behavior continues to $\tan \beta \sim 40-50$. Then additional Yukawa couplings also play a significant role at larger $\tan \beta$ and reduce fine-tuning further by improving the radiative EW symmetry breaking for the same $m_{h}$ (this is because radiative EW symmetry breaking effects are enhanced relative to opposite, QCD ones that increase fine-tuning [38-41]). We thus expect that for the case of large $\tan \beta$ with additional Yukawa couplings included the values quoted here for $\Delta_{m}$, $\Delta_{q}$ be maintained or reduced further.

Unlike other attempts to reduce the EW fine-tuning, the present case has the advantage that it does not introduce new states in the visible sector. However, there still is a "cost"

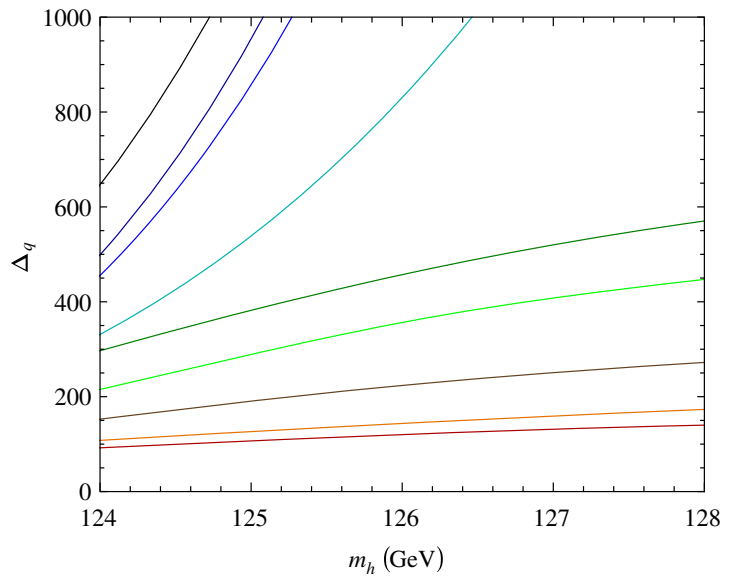

3.9 TeV (brown), $5 \mathrm{TeV}$ (green), 5.5 TeV (dark green), 6.3 TeV (cyan), $7.4 \mathrm{TeV}$ (blue), $8 \mathrm{TeV}$ (dark blue), $8.7 \mathrm{TeV}$ (black/top curve). The lowest two curves (red, orange) correspond to the minimal values of $\Delta_{m}$ and $\Delta_{q}$ in Figs. 2 and 3. For large enough $\sqrt{f} \geq 10 \mathrm{TeV}$, one recovers the MSSM-like values of $\Delta_{m}, \Delta_{q}$ for a similar $m_{h}$

at the phenomenological level. In models with a TeV scale for SUSY breaking, the gravitino is very light (milli-eV) and the usual MSSM-like account for dark matter (as due to the LSP) cannot apply. This is a standard problem for models with a low scale of SUSY breaking, and alternative dark matter candidates need to be considered (the axino [76], or the axion [77]; for a review see [78]).

\section{Conclusions}

The significant amount of EW fine-tuning $\Delta$ present in the MSSM-like models for $m_{h} \approx 126 \mathrm{GeV}$ has prompted an increased interest in finding ways to reduce its value. This is motivated by the fact that $\Delta$ is usually regarded as a measure of the success of SUSY in solving the hierarchy problem. Additional reasons to seek a low $\Delta$ exist, from the relation of the EW fine-tuning to the variation $\delta \chi^{2}$ about the minimal chi-square $\chi_{\min }^{2}$ and the s-standard deviation upper bound on $\delta \chi^{2}$ usually sought in the data fits. Reducing $\Delta$ can indeed be achieved, but it usually requires the introduction of additional fields in the visible sector, beyond those of the original model. For example, one can consider MSSM-like models with additional, massive gauge singlets present, extra gauge symmetries, etc.

Another point of view is that a large EW fine-tuning may indicate a problem with our understanding of supersymmetry breaking. Motivated by this we considered the case of MSSM-like models with a low scale of supersymmetry breaking in the hidden sector, $\sqrt{f} \sim$ few TeV. As a result of this, sizeable quartic effective interactions are present in the Higgs potential, generated by the exchange of the auxiliary field of the goldstino superfield. Such couplings are 
proportional to the ratio of the soft breaking terms $m_{\text {soft in }}$ the visible sector to the SUSY breaking scale $\sqrt{f}$ of the hidden sector. Thus, such couplings are significant in models with $\sqrt{f} \sim$ few TeV and are negligible when $\sqrt{f}$ is large, which is the usual MSSM scenario. These couplings have significant implications for the Higgs mass and the EW finetuning. This behavior is generic in low-scale SUSY models.

For the most conservative case of a constrained "nonlinear" MSSM model and at low $\tan \beta$, we computed the level of EW scale fine-tuning measured by two definitions for $\Delta$ $\left(\Delta_{m}, \Delta_{q}\right)$. We examined $\Delta_{m, q}$ as a function of the SM-like Higgs mass, in the one-loop approximation for these quantities. The results show that for $m_{h} \approx 126 \mathrm{GeV}$, fine-tuning is reduced from minimal values of $\approx 800-1000$ in the constrained MSSM to more acceptable values of $\sim 80-100$ in our model with $\sqrt{f} \sim 2.8-3.2 \mathrm{TeV}$. These values for $\Delta$ are expected to be further reduced by considering non-universal gaugino masses. We argued that a similar reduction of $\Delta$ is expected at large $\tan \beta$ in our model. For larger $\sqrt{f}$, usually above $10 \mathrm{TeV}$, one recovers the case of MSSM-like models. Unlike other similar studies, the reduction of $\Delta$ was possible without additional fields in the visible sector and depends only on the ratio(s) $m_{\text {soft }}^{2} / f$. One may consider the intriguing possibility of increasing simultaneously one of the soft masses $m_{\text {soft }}$ (say $m_{0}$ ) and $\sqrt{f}$, with their ratio fixed (this could keep unchanged the leading corrections $\mathcal{O}\left[\left(m_{\text {soft }}^{2} / f\right)^{2}\right]$ for the Higgs mass and $\Delta$ ). This is relevant if no superpartners are found near the $\mathrm{TeV}$ scale.

We assumed that in our case the sgoldstino was massive enough and integrated out, by using the superfield constraint that decouples it from the low energy. Corrections to our result can then arise from the scalar potential for the sgoldstino that depends on the structure of its Kähler potential (which gives mass to it) and the superpotential in the hidden sector. Another correction can arise from future experimental constraints that may increase the lower bounds on the value of $\sqrt{f}$, currently near $\approx 700 \mathrm{GeV}$, if no supersymmetry or other new physics signal is found.

Acknowledgments This work was supported in part by the European Commission under the ERC Advanced Grant 226371.

The work of D. M. Ghilencea was supported by a grant from the Romanian National Authority for Scientific Research, CNCS-UEFISCDI, Project Number PN-II-ID-PCE-2011-3-0607 and in part by the National Programme 'Nucleu' PN 093701 02. The work of E. M. Babalic was supported by strategic grant POSDRU/159/1.5/S/133255, (project ID 133255/2014), co-financed by the European Social Fund within the Sectorial Operational Program Human Resources Development 20072013; CNCS-UEFISCDI grant PN-II-ID-PCE 121/2011 and PN 0937 0102.

Open Access This article is distributed under the terms of the Creative Commons Attribution License which permits any use, distribution, and reproduction in any medium, provided the original author(s) and the source are credited.

Funded by $\mathrm{SCOAP}^{3}$ / License Version CC BY 4.0.

\section{Appendix}

The coefficients $\sigma_{i}$ at the EW scale, used in the text, Eq. (28), have the following values

$$
\begin{aligned}
& \sigma_{1}\left(t_{z}\right)=0.532, \sigma_{2}\left(t_{z}\right)=0.282\left(4.127 h_{t}^{2}-2.783\right) \\
& \quad\left(1.310-h_{t}^{2}\right)^{1 / 4}, \\
& \sigma_{3}\left(t_{z}\right)=-0.501 h_{t}^{2}\left(1.310-h_{t}^{2}\right)^{1 / 4}, \\
& \sigma_{4}\left(t_{z}\right)=0.532-5.233 h_{t}^{2}+1.569 h_{t}^{4}, \\
& \sigma_{5}\left(t_{z}\right)=0.125 h_{t}^{2}\left(10.852 h_{t}^{2}-14.221\right), \\
& \sigma_{6}\left(t_{z}\right)=-0.027 h_{t}^{2}\left(10.852 h_{t}^{2}-14.221\right), \\
& \sigma_{7}\left(t_{z}\right)=1-1.145 h_{t}^{2}, \sigma_{8}\left(t_{z}\right)=1.314\left(1.310-h_{t}^{2}\right)^{1 / 4}
\end{aligned}
$$

where $h_{t}$ is evaluated at $m_{Z}$ and $m_{t}=h_{t}\left(t_{m_{t}}\right)(v / \sqrt{2}) \sin \beta$ $(v=246 \mathrm{GeV}), t=\ln \Lambda^{2} / q^{2}, t_{z}=\ln \Lambda_{U V}^{2} / m_{Z}^{2}$.

\section{References}

1. P. Fayet, Mixing between gravitational and weak interactions through the massive gravitino. Phys. Lett. B 70, 461 (1977)

2. P. Fayet, Weak interactions of a light gravitino: a lower limit on the gravitino mass from the decay psi $\rightarrow$ gravitino anti-photino. Phys. Lett. B 84, 421 (1979)

3. P. Fayet, Scattering cross-sections of the photino and the goldstino (gravitino) on matter. Phys. Lett. B 86, 272 (1979)

4. R. Casalbuoni, S. De Curtis, D. Dominici, F. Feruglio, R. Gatto, A gravitino-goldstino high-energy equivalence theorem. Phys. Lett. B 215, 313 (1988)

5. R. Casalbuoni, S. De Curtis, D. Dominici, F. Feruglio, R. Gatto, High-energy equivalence theorem in spontaneously broken supergravity. Phys. Rev. D 39, 2281 (1989)

6. I. Antoniadis, E. Dudas, D.M. Ghilencea, P. Tziveloglou, Nonlinear MSSM. Nucl. Phys. B 841, 157 (2010). arXiv:1006.1662 [hep-ph]

7. C. Petersson, A. Romagnoni, The MSSM Higgs sector with a dynamical goldstino supermultiplet. JHEP 1202, 142 (2012). arXiv:1111.3368 [hep-ph]

8. Z. Komargodski, N. Seiberg, From linear SUSY to constrained superfields. JHEP 0909, 066 (2009). arXiv:0907.2441 [hep-th]

9. R. Casalbuoni, S. De Curtis, D. Dominici, F. Feruglio, R. Gatto, Nonlinear realization of supersymmetry algebra from supersymmetric constraint. Phys. Lett. B 220, 569 (1989)

10. M. Rocek, Linearizing the Volkov-Akulov model. Phys. Rev. Lett. 41, 451 (1978)

11. U. Lindstrom, M. Rocek, Constrained local superfields. Phys. Rev. D 19, 2300 (1979)

12. E. Dudas, G. von Gersdorff, D.M. Ghilencea, S. Lavignac, J. Parmentier, On non-universal goldstino couplings to matter. Nucl. Phys. B 855, 570 (2012). arXiv:1106.5792 [hep-th]

13. I. Antoniadis, D.M. Ghilencea, Low-scale SUSY breaking and the (s)goldstino physics. Nucl. Phys. B 870, 278 (2013). arXiv:1210.8336 [hep-th]

14. I. Antoniadis, E. Dudas, D.M. Ghilencea, Goldstino and sgoldstino in microscopic models and the constrained superfields formalism. Nucl. Phys. B 857, 65 (2012). arXiv:1110.5939 [hep-th] 
15. S. Demidov, K. Astapov, Implications of sgoldstino-Higgs mixing. PoS QFTHEP 2013, 090 (2014)

16. D.S. Gorbunov, V.A. Rubakov, On sgoldstino interpretation of HyperCP events. Phys. Rev. D 73, 035002 (2006). hep-ph/0509147

17. J.R. Ellis, K. Enqvist, D.V. Nanopoulos, F. Zwirner, Observables in low-energy superstring models. Mod. Phys. Lett. A 1, 57 (1986)

18. R. Barbieri, G.F. Giudice, Upper bounds on supersymmetric particle masses. Nucl. Phys. B 306, 63 (1988)

19. G.W. Anderson, D.J. Castano, Measures of fine tuning. Phys. Lett. B 347, 300 (1995). hep-ph/9409419

20. G.W. Anderson, D.J. Castano, Naturalness and superpartner masses or when to give up on weak scale supersymmetry. Phys. Rev. D 52, 1693 (1995). hep-ph/9412322

21. R. Barbieri, A. Strumia, About the fine tuning price of LEP. Phys. Lett. B 433, 63 (1998). hep-ph/9801353

22. P.H. Chankowski, J.R. Ellis, M. Olechowski, S. Pokorski, Haggling over the fine tuning price of LEP. Nucl. Phys. B 544, 39 (1999). hep-ph/9808275

23. G.L. Kane, S.F. King, Naturalness implications of LEP results. Phys. Lett. B 451, 113 (1999). hep-ph/9810374

24. P.H. Chankowski, J.R. Ellis, S. Pokorski, The fine tuning price of LEP. Phys. Lett. B 423, 327 (1998). hep-ph/9712234

25. G.F. Giudice, R. Rattazzi, Living dangerously with low-energy supersymmetry. Nucl. Phys. B 757, 19 (2006). hep-ph/0606105

26. G. Aad et al. [ATLAS Collaboration], Observation of a new particle in the search for the Standard Model Higgs boson with the ATLAS detector at the LHC. Phys. Lett. B 716, 1 (2012). arXiv:1207.7214 [hep-ex]

27. S. Chatrchyan et al. [CMS Collaboration], Observation of a new boson at a mass of $125 \mathrm{GeV}$ with the CMS experiment at the LHC. Phys. Lett. B 716, 30 (2012). arXiv:1207.7235 [hep-ex]

28. G. Aad et al. [ATLAS Collaboration], Report ATLASCONF-2012-162, Updated ATLAS results on the signal strength of the Higgs-like boson for decays into WW and heavy fermion final states, Nov. 2012 (2012)

29. S. Chatrchyan et al. [CMS Collaboration], Report CMS-PAS-HIG12-045, Combination of standard model Higgs boson searches and measurements of the properties of the new boson with a mass near $125 \mathrm{GeV}, 23$ Nov 2012 (2012)

30. D.M. Ghilencea, H.M. Lee, M. Park, Tuning supersymmetric models at the LHC: a comparative analysis at 2-loop level. JHEP 1207, 046 (2012). arXiv:1203.0569 [hep-ph]

31. D.M. Ghilencea, G.G. Ross, The fine-tuning cost of the likelihood in SUSY models. Nucl. Phys. B 868, 65 (2013). arXiv:1208.0837 [hep-ph]

32. D.M. Ghilencea, Fixing the EW scale in supersymmetric models after the Higgs discovery. Nucl. Phys. B 876, 16 (2013). arXiv: 1302.5262 [hep-ph]

33. D.M. Ghilencea, A new approach to naturalness in SUSY models. PoS Corfu 2012, 034 (2013). arXiv:1304.1193 [hep-ph]

34. B.C. Allanach, K. Cranmer, C.G. Lester, A.M. Weber, Natural priors. CMSSM fits and LHC weather forecasts. JHEP 0708, 023 (2007). arXiv:0705.0487 [hep-ph]

35. M.E. Cabrera, J.A. Casas, R. Ruiz de Austri, Bayesian approach and naturalness in MSSM analyses for the LHC. JHEP 0903, 075 (2009). arXiv:0812.0536 [hep-ph]

36. M.E. Cabrera, J.A. Casas, R. Ruiz d Austri, MSSM forecast for the LHC. JHEP 1005, 043 (2010). arXiv:0911.4686 [hep-ph]

37. S.S. AbdusSalam, B.C. Allanach, F. Quevedo, F. Feroz, M. Hobson, Fitting the phenomenological MSSM. Phys. Rev. D 81, 095012 (2010). arXiv:0904.2548 [hep-ph]

38. S. Cassel, D.M. Ghilencea, G.G. Ross, Testing SUSY at the LHC: electroweak and dark matter fine tuning at two-loop order. Nucl. Phys. B 835, 110 (2010). arXiv:1001.3884 [hep-ph]
39. S. Cassel, D.M. Ghilencea, G.G. Ross, Testing SUSY. Phys. Lett. B 687, 214 (2010). arXiv:0911.1134 [hep-ph]

40. S. Cassel, D.M. Ghilencea, S. Kraml, A. Lessa, G.G. Ross, Finetuning implications for complementary dark matter and LHC SUSY searches. JHEP 1105, 120 (2011). arXiv:1101.4664 [hep$\mathrm{ph}]$

41. S. Cassel, D.M. Ghilencea, A review of naturalness and dark matter prediction for the Higgs mass in MSSM and beyond. Mod. Phys. Lett. A 27, 1230003 (2012). arXiv:1103.4793 [hep-ph]

42. B.C. Allanach, SOFTSUSY: a program for calculating supersymmetric spectra. Comput. Phys. Commun. 143, 305 (2002). hep-ph/0104145

43. G. Degrassi, S. Heinemeyer, W. Hollik, P. Slavich, G. Weiglein, Towards high precision predictions for the MSSM Higgs sector. Eur. Phys. J. C 28, 133 (2003). hep-ph/0212020

44. S. Heinemeyer, MSSM Higgs physics at higher orders. Int. J. Mod. Phys. A 21, 2659 (2006). hep-ph/0407244

45. P. Bechtle, T. Bringmann, K. Desch, H. Dreiner, M. Hamer, C. Hensel, M. Kramer, N. Nguyen et al., Constrained supersymmetry after two years of LHC data: a global view with Fittino. JHEP 1206, 098 (2012). arXiv:1204.4199 [hep-ph]

46. D.M. Ghilencea, SUSY naturalness without prejudice. Phys. Rev. D 89, 095007 (2014). arXiv:1311.6144 [hep-ph]

47. J.A. Casas, J.R. Espinosa, I. Hidalgo, The MSSM fine tuning problem: a way out. JHEP 0401, 008 (2004). arXiv:hep-ph/0310137

48. J.A. Casas, J.R. Espinosa, I. Hidalgo, A relief to the supersymmetric fine tuning problem. arXiv:hep-ph/0402017

49. A. Brignole, J.A. Casas, J.R. Espinosa, I. Navarro, Low-scale supersymmetry breaking: effective description, electroweak breaking and phenomenology. Nucl. Phys. B 666, 105 (2003). arXiv:hep-ph/0301121

50. G.G. Ross, K. Schmidt-Hoberg, The fine-tuning of the generalised NMSSM. Nucl. Phys. B 862, 710 (2012). arXiv:1108.1284 [hep$\mathrm{ph}]$

51. G.G. Ross, K. Schmidt-Hoberg, F. Staub, The generalised NMSSM at one loop: fine tuning and phenomenology. JHEP 1208, 074 (2012). arXiv:1205.1509 [hep-ph]. See also [31]

52. M.T. Grisaru, M. Rocek, A. Karlhede, The superHiggs effect in superspace. Phys. Lett. B 120, 110 (1983)

53. E. Cremmer, B. Julia, J. Scherk, P. van Nieuwenhuizen, S. Ferrara, L. Girardello, SuperHiggs effect in supergravity with general scalar interactions. Phys. Lett. B 79, 231 (1978)

54. D.V. Volkov, V.P. Akulov, Is the neutrino a goldstone particle? Phys. Lett. B 46, 109 (1973)

55. T.E. Clark, S.T. Love, Goldstino couplings to matter. Phys. Rev. D 54, 5723 (1996). arXiv:hep-ph/9608243

56. T.E. Clark, T. Lee, S.T. Love, G. Wu, On the interactions of light gravitinos. Phys. Rev. D 57, 5912 (1998). arXiv:hep-ph/9712353

57. A. Brignole, F. Feruglio, F. Zwirner, On the effective interactions of a light gravitino with matter fermions. JHEP 9711, 001 (1997). arXiv:hep-th/9709111

58. M.A. Luty, E. Ponton, Effective Lagrangians and light gravitino phenomenology. Phys. Rev. D 57, 4167 (1998). arXiv:hep-ph/9706268, v3 [revised version of Phys. Rev. D 57, 4167 (1998)]

59. E.A. Ivanov, A.A. Kapustnikov, General relationship between linear and nonlinear realizations of supersymmetry. J. Phys. A 11, 2375 (1978)

60. E.A. Ivanov, A.A. Kapustnikov, The nonlinear realization structure of models with spontaneously broken supersymmetry. J. Phys. G 8, 167 (1982)

61. S. Samuel, J. Wess, A superfield formulation of the nonlinear realization of supersymmetry and its coupling to supergravity. Nucl. Phys. B 221, 153 (1983) 
62. S. Cassel, D.M. Ghilencea, G.G. Ross, Fine tuning as an indication of physics beyond the MSSM. Nucl. Phys. B 825, 203 (2010). arXiv:0903.1115 [hep-ph]

63. M. Carena, K. Kong, E. Ponton, J. Zurita, Supersymmetric Higgs bosons and beyond. Phys. Rev. D 81, 015001 (2010). arXiv:0909.5434

64. M. Carena, E. Ponton, J. Zurita, BMSSM Higgs bosons at the Tevatron and the LHC. Phys. Rev. D 82, 055025 (2010). arXiv:1005.4887 [hep-ph]

65. I. Antoniadis, E. Dudas, D.M. Ghilencea, P. Tziveloglou, MSSM Higgs with dimension-six operators. Nucl. Phys. B 831, 133 (2010). arXiv:0910.1100 [hep-ph]

66. I. Antoniadis, E. Dudas, D.M. Ghilencea, P. Tziveloglou, Beyond the MSSM Higgs with $\mathrm{d}=6$ effective operators. Nucl. Phys. B 848, 1 (2011). arXiv:1012.5310 [hep-ph]

67. I. Antoniadis, E. Dudas, D.M. Ghilencea, P. Tziveloglou, MSSM with dimension-five operators $\left(\mathrm{MSSM}_{5}\right)$. Nucl. Phys. B 808, 155 (2009). arXiv:0806.3778 [hep-ph]

68. I. Antoniadis, E. Dudas, D.M. Ghilencea, Supersymmetric models with higher dimensional operators. JHEP 0803, 045 (2008). arXiv:0708.0383 [hep-th]

69. M. Dine, N. Seiberg, S. Thomas, Higgs physics as a window beyond the MSSM (BMSSM). Phys. Rev. D 76, 095004 (2007). arXiv:0707.0005 [hep-ph]

70. I. Antoniadis, M. Tuckmantel, F. Zwirner, Phenomenology of a leptonic goldstino and invisible Higgs boson decays. Nucl. Phys. B 707, 215 (2005). arXiv:hep-ph/0410165
71. H. Baer, Radiative natural supersymmetry with mixed axion/higgsino cold dark matter. AIP Conf. Proc. 1534, 39 (2012). arXiv:1210.7852 [hep-ph]

72. H. Baer, V. Barger, P. Huang, A. Mustafayev, X. Tata, Radiative natural SUSY with a $125 \mathrm{GeV}$ Higgs boson. Phys. Rev. Lett. 109, 161802 (2012). arXiv: 1207.3343 [hep-ph]

73. H. Baer, V. Barger, D. Mickelson, M. Padeffke-Kirkland, SUSY models under siege: LHC constraints and electroweak fine-tuning. arXiv: 1404.2277 [hep-ph]

74. D. Horton, G.G. Ross, Naturalness and focus points with nonuniversal gaugino masses. Nucl. Phys. B 830, 221 (2010). arXiv:0908.0857 [hep-ph]

75. A. Kaminska, G.G. Ross, K. Schmidt-Hoberg, Non-universal gaugino masses and fine tuning implications for SUSY searches in the MSSM and the GNMSSM. JHEP 1311, 209 (2013). arXiv: 1308.4168 [hep-ph]

76. L. Covi, H.B. Kim, J.E. Kim, L. Roszkowski, Axinos as dark matter. JHEP 0105, 033 (2001). hep-ph/0101009

77. H. Baer, S. Kraml, A. Lessa, S. Sekmen, Thermal leptogenesis and the gravitino problem in the Asaka-Yanagida axion/axino dark matter scenario. JCAP 1104, 039 (2011). arXiv:1012.3769 [hep$\mathrm{ph}]$

78. J.L. Feng, Dark matter candidates from particle physics and methods of detection. Ann. Rev. Astron. Astrophys. 48, 495 (2010). arXiv:1003.0904 [astro-ph.CO] 